\title{
Nonstationary Stokes system in Sobolev-Slobodetski spaces
}

\author{
Wojciech M. Zajączkowski
}

Received: 15 April 2011 / Revised: 15 February 2012 / Published online: 9 September 2012 (C) The Author(s) 2012. This article is published with open access at Springerlink.com

\begin{abstract}
We consider an initial-boundary value problem for the nonstationary Stokes system in a bounded domain $\Omega \subset \mathbb{R}^{3}$ with slip boundary conditions. We prove the existence in the Hilbert-Sobolev-Slobodetski spaces with fractional derivatives. The proof is divided into two main steps. In the first step by applying the compatibility conditions an extension of initial data transforms the considered problem to a problem with vanishing initial data such that the right-hand sides data functions can be extended by zero on the negative half-axis of time in the above mentioned spaces. The problem with vanishing initial data is transformed to a functional equation by applying an appropriate partition of unity. The existence of solutions of the equation is proved by a fixed point theorem. We prove the existence of such solutions that $v \in H^{l+2, l / 2+1}(\Omega \times(0, T)), \nabla p \in H^{l, l / 2}(\Omega \times(0, T)), v$-velocity, $p$-pressure, $l \in \mathbb{R}_{+} \cup\{0\}, l \neq[l]+\frac{1}{2}$ and the spaces are introduced by Slobodetski and used extensively by Lions-Magenes. We should underline that to show solvability of the Stokes system we need only solvability of the heat and the Poisson equations in $\mathbb{R}^{3}$ and $\mathbb{R}_{+}^{3}$. This is possible because the slip boundary conditions are considered.
\end{abstract}

Mathematics Subject Classification (2000) $\quad 35 \mathrm{Q} 30 \cdot 76 \mathrm{D} 07 \cdot 76 \mathrm{~B} 03$

The author is partially supported by Polish Grant No NN 201396937.

W. M. Zajączkowski ( $\varangle)$

Institute of Mathematics, Polish Academy of Sciences,

Śniadeckich 8, 00-956 Warsaw, Poland

e-mail:wz@impan.pl

W. M. Zajączkowski

Institute of Mathematics and Cryptology, Cybernetics Faculty,

Military University of Technology,

Kaliskiego 2, 00-908 Warsaw, Poland 


\section{Introduction}

The aim of this paper is solvability in Sobolev-Slobodetski spaces of the Hilbert type of the following slip boundary value problem for the nonstationary Stokes system

$$
\begin{aligned}
& v_{t}-\operatorname{div} \mathbb{T}(v, p)=f_{0} \\
& \operatorname{div} v=g_{0} \\
& \text { in } \Omega^{T}=\Omega \times(0, T) \text {, } \\
& \text { in } \Omega^{T} \text {, } \\
& \nu \bar{n} \cdot \mathbb{D}(v) \cdot \bar{\tau}_{\alpha}+\gamma v \cdot \bar{\tau}_{\alpha}=h_{0 \alpha}, \quad \alpha=1,2, \quad \text { on } \quad S^{T}=S \times(0, T) \text {, } \\
& v \cdot \bar{n}=h_{03} \\
& \text { on } S^{T} \text {, } \\
& \left.v\right|_{t=0}=v_{0} \\
& \text { in } \Omega \text {, } \\
& \int_{\Omega} p d x=0 \text {, }
\end{aligned}
$$

where $\Omega \subset \mathbb{R}^{3}, S$ is the boundary of $\Omega, v=\left(v_{1}(x, t), v_{2}(x, t), v_{3}(x, t)\right) \in \mathbb{R}^{3}$ is the velocity of the fluid, $x=\left(x_{1}, x_{2}, x_{3}\right)$ the Cartesian system in $\mathbb{R}^{3}, f_{0}=$ $\left(f_{01}(x, t), f_{02}(x, t), f_{03}(x, t)\right) \in \mathbb{R}^{3}$ the external force field, $p=p(x, t) \in \mathbb{R}$ the pressure. Moreover, $\bar{n}$ is the unit outward vector normal to $S, \bar{\tau}_{\alpha}, \alpha=1,2$, tangent to $S, \gamma \geq 0$ is the constant slip coefficient.

By $\mathbb{T}(v, p)$ we denote the stress tensor of the form

$$
\mathbb{T}(v, p)=v \mathbb{D}(v)-p \mathbb{I},
$$

where $v>0$ is the constant viscosity coefficient, $\mathbb{I}$ is the unit matrix and $\mathbb{D}(v)$ is the dilatation tensor of the form

$$
\mathbb{D}(v)=\left\{v_{i, x_{j}}+v_{j, x_{i}}\right\}_{i, j=1,2,3}=\nabla v+(\nabla v)^{T} .
$$

To formulate the main result of this paper we need.

Definition 1.1 (see equivalent Definition 2.1) By $W_{2}^{l, l / 2}\left(Q^{T}\right), l \in \mathbb{R}_{+} \cup\{0\}, Q \subset$ $\mathbb{R}^{n}$, we define the anisotropic Sobolev-Slobodetski space as a set of functions with the following finite norm

$$
\begin{aligned}
& \|u\|_{W_{2}^{l, l / 2}\left(Q^{T}\right)}=\left(\sum_{|\alpha|+2 a \leq l} \int_{Q^{T}}\left|D_{x}^{\alpha} \partial_{t}^{a} u\right|^{2} d x d t\right. \\
& +\sum_{|\alpha|+2 a=[l]} \int_{0}^{T} \int_{Q} \int_{Q} \frac{\left|D_{x}^{\alpha} \partial_{t}^{a} u(x, t)-D_{x^{\prime}}^{\alpha} \partial_{t}^{a} u\left(x^{\prime}, t\right)\right|^{2}}{\left|x-x^{\prime}\right|^{n+2(l-[l])} d x d x^{\prime} d t} \\
& +\sum_{|\alpha|+2 a=[l]} \int_{Q}^{T} \int_{0}^{T} \frac{\left|D_{x}^{\alpha} \partial_{t}^{a} u(x, t)-D_{x}^{\alpha} \partial_{t^{\prime}}^{a} u\left(x, t^{\prime}\right)\right|^{2}}{\left.\left|t-t^{\prime}\right|^{1+2(l / 2-[l / 2])} d x d t d t^{\prime}\right)^{1 / 2}} .
\end{aligned}
$$

In the case of integer $l$ the middle integral and for $l$ even the last two integrals disappear. 
To simplify notation we introduce $H^{l, l / 2}\left(Q^{T}\right)=W_{2}^{l, l / 2}\left(Q^{T}\right)$. Let $Q$ be a submanifold of $\mathbb{R}^{n}$. Then norm (1.3) is defined by an appropriate partition of unity.

To prove the existence of solutions to problem (1.1) in $H^{s+2, s / 2+1}\left(\Omega^{T}\right), s \in \mathbb{R}_{+} \cup$ $\{0\}$, we need compatibility conditions.

Definition 1.2 Assume the compatibility conditions

$$
\begin{aligned}
\int_{\Omega} g_{0} d x & =\int_{S} h_{03} d S . \\
D_{x}^{\alpha} \operatorname{div} v_{0} & =\left.D_{x}^{\alpha} g_{0}\right|_{t=0}, \quad|\alpha| \leq s-1,
\end{aligned}
$$

where $D_{x}^{\alpha}=\partial_{x_{1}}^{\alpha_{1}} \partial_{x_{2}}^{\alpha_{2}} \partial_{x_{3}}^{\alpha_{3}},|\alpha|=\alpha_{1}+\alpha_{2}+\alpha_{3}, \alpha=\left(\alpha_{1}, \alpha_{2}, \alpha_{3}\right)$ is the multiindex.

$$
\begin{aligned}
& D_{\tau}^{\beta}\left(\nu \bar{n} \cdot \mathbb{D}\left(v_{0}\right) \cdot \bar{\tau}_{\alpha}+\gamma v_{0} \cdot \bar{\tau}_{\alpha}\right)=\left.D_{\tau}^{\beta} h_{0 \alpha}\right|_{t=0}, \quad \alpha=1,2, \text { on } S, \\
& |\beta| \leq s-3 / 2, \quad \beta=\left(\beta_{1}, \beta_{2}\right), \quad|\beta|=\beta_{1}+\beta_{2}, \quad D_{\tau}^{\beta}=\partial_{\tau_{1}}^{\beta_{1}} \partial_{\tau_{2}}^{\beta_{2}},
\end{aligned}
$$

$\partial_{\tau_{\alpha}}$ is the tangent derivative to $S$ directed along the tangent vector $\bar{\tau}_{\alpha}, \alpha=1,2$

$$
\begin{aligned}
D_{\tau}^{\beta} v_{0} \cdot \bar{n} & =\left.D_{\tau}^{\beta} h_{03}\right|_{t=0}, \quad|\beta| \leq s-1 / 2, \\
\int_{\Omega} p d x & =0 .
\end{aligned}
$$

A very important step in the existence theory of solutions to the nonstationary problem (1.1) is its transformation to a problem with time $t \in \mathbb{R}$. For this purpose we transform problem (1.1) to a problem with vanishing initial data by its appropriate extension.

Next such compatibility conditions on the r.h.s. functions in Eq. (1.1) $1,2,3,4$ must be assumed that after the above extension of the initial data they can be extended by zero for $t<0$ in the considered spaces.

For this we extend the initial data in such a way that $\left.\tilde{v}_{0}\right|_{t=0}=v_{0}, \tilde{v}_{0} \in$ $H^{2+s, 1+s / 2}\left(\Omega^{T}\right)$ and

$$
\left\|\tilde{v}_{0}\right\|_{H^{2+s, 1+s / 2}\left(\Omega^{T}\right)} \leq c\left\|v_{0}\right\|_{H^{1+s}(\Omega)} .
$$

Then we impose the following compatibility conditions

Definition 1.3 Assume that $\tilde{v}, f_{0}, g_{0}, h_{0}$ satisfy the relations

$$
\begin{aligned}
& \left.\partial_{t}^{\sigma}\left(f_{0}-\tilde{v}_{0, t}+v \operatorname{div} \mathbb{D}\left(\tilde{v}_{0}\right)\right)\right|_{t=0}=0, \\
& \sigma<\frac{s}{2} \text { for } \frac{s}{2} \notin \mathbb{Z}, \quad \sigma \leq \frac{s}{2}-1 \text { for } \frac{s}{2} \in \mathbb{Z} ; \\
& \left.\partial_{t}^{\sigma}\left(g_{0}-\operatorname{div} \tilde{v}_{0}\right)\right|_{t=0}=0, \\
& \sigma<\frac{s+1}{2} \text { for } \frac{s+1}{2} \notin \mathbb{Z}, \quad \sigma \leq \frac{s+1}{2}-1 \text { for } \frac{s+1}{2} \in \mathbb{Z} ; \\
& \left.\partial_{t}^{\sigma}\left(h_{0 \alpha}-v \bar{n} \cdot \mathbb{D}\left(\tilde{v}_{0}\right) \cdot \bar{\tau}_{\alpha}-\gamma \tilde{v}_{0} \cdot \bar{\tau}_{\alpha}\right)\right|_{t=0}=0, \quad \alpha=1,2,
\end{aligned}
$$




$$
\begin{aligned}
& \sigma<\frac{s+1 / 2}{2} \text { for } \frac{s+1 / 2}{2} \notin \mathbb{Z}, \quad \sigma \leq \frac{s+1 / 2}{2}-1 \text { for } \frac{s+1 / 2}{2} \in \mathbb{Z} ; \\
& \left.\partial_{t}^{\sigma}\left(h_{03}-\tilde{v}_{0} \cdot \bar{n}\right)\right|_{t=0}=0, \\
& \sigma<\frac{3}{4}+\frac{s}{2} \text { for } \frac{3}{4}+\frac{s}{2} \notin \mathbb{Z}, \quad \sigma \leq \frac{3}{4}+\frac{s}{2}-1 \text { for } \frac{3}{4}+\frac{s}{2} \in \mathbb{Z} .
\end{aligned}
$$

Definition 1.4 (see [1, Proposition 1.7]) Assume that $s / 2 \neq k+1 / 2, k \in \mathbb{Z}_{+} \cup\{0\}$.

The main result of this paper reads

Theorem A Assume that $S \in C^{s+2}, f_{0} \in H^{s, s / 2}\left(\Omega^{T}\right), g_{0} \in H^{s / 2+1, s / 2}\left(\Omega^{T}\right), g_{0, t} \in$ $L_{2}\left(\Omega ; H^{s / 2}(0, T)\right), h_{0}^{\prime}=\left(h_{01}, h_{02}\right) \in H^{s+1 / 2, s / 2+1 / 4}\left(S^{T}\right), h_{03} \in H^{s+3 / 2, s / 2+3 / 4}$ $\left(S^{T}\right), v_{0} \in H^{1+s}(\Omega), s \in \mathbb{R}_{+} \cup\{0\}$. Assume the compatibility conditions introduced in Definitions 1.2, 1.3, 1.4. Then there exists a solution to problem (1.1) such that $v \in H^{s+2, s / 2+1}\left(\Omega^{T}\right), \nabla p \in H^{s, s / 2}\left(\Omega^{T}\right)$ and there exists a constant $c_{0}$ depending at most on $\|S\|_{C^{s+2}}, s, T$, such that

$$
\begin{aligned}
& \|v\|_{H^{2+s, 1+s / 2}\left(\Omega^{T}\right)}+\|\nabla p\|_{H^{s, s / 2}\left(\Omega^{T}\right)} \\
& \leq c_{0}\left(\left\|f_{0}\right\|_{H^{s, s / 2}\left(\Omega^{T}\right)}+\left\|g_{0}\right\|_{H^{s+1, s / 2}\left(\Omega^{T}\right)}\right. \\
& \quad+\left\|g_{0 t}\right\|_{L_{2}\left(\Omega ; H^{s / 2}(0, T)\right)}+\left\|h_{0}^{\prime}\right\|_{H^{s+1 / 2, s / 2+1 / 4}\left(S^{T}\right)} \\
& \left.\quad+\left\|h_{03}\right\|_{H^{s+3 / 2, s / 2+3 / 4}\left(S^{T}\right)}+\left\|v_{0}\right\|_{H^{s+1}(\Omega)}\right) .
\end{aligned}
$$

For $c_{0}$ independent of $T$ see Sect. 6.

Remark 1.5 To describe the compatibility conditions (1.10) we have first to construct function $\tilde{v}_{0}$. From (1.10) the following iterative relation follows

$$
\left.\partial_{t}^{k} \tilde{v}_{0}\right|_{t=0}=\left.\partial_{t}^{k-1} f_{0}\right|_{t=0}+v \operatorname{div} \mathbb{D}\left(\left.\partial_{t}^{k-1} \tilde{v}_{0}\right|_{t=0}\right)
$$

where $k<\frac{s}{2}+1$ for $\frac{s}{2} \notin \mathbb{Z}$ and $k \leq \frac{s}{2}$ for $\frac{s}{2} \in \mathbb{Z}$. Since

$$
\left.\tilde{v}_{0}\right|_{t=0}=v_{0}
$$

for $k=1$ we have

$$
\left.\partial_{t} \tilde{v}_{0}\right|_{t=0}=\left.f_{0}\right|_{t=0}+v \operatorname{div} \mathbb{D}\left(v_{0}\right) .
$$

Calculating $\left.\partial_{t}^{k} \tilde{v}_{0}\right|_{t=0}$ from (1.12) and (1.13) step by step we obtain

$$
\left.\partial_{t}^{k} \tilde{v}_{0}\right|_{t=0}=b^{(k)}\left(\left.f_{0}\right|_{t=0}, v_{0}\right)
$$

where $b^{(k)}$ is a differential operator with respect to space variables of order $2 k$ for $v_{0}$ and $2 k-2$ for $\left.f_{0}\right|_{t=0}$, and $k$ is described above. 
From (1.13), (1.15) there exists a function $\tilde{v}_{0} \in H^{s+2, s / 2+1}\left(\Omega^{T}\right)$ such that (1.13), (1.15) are satisfied for all $k$ defined above and

$$
\left\|\tilde{v}_{0}\right\|_{H^{s+2, s / 2+1}\left(\Omega^{T}\right)} \leq c \sum_{k=0}^{\left[\frac{s}{2}+1\right]}\left\|b^{(k)}\left(\left.f_{0}\right|_{t=0}, v_{0}\right)\right\|_{H^{s+2-2 k}(\Omega)},
$$

where $b^{0}=v_{0}$.

Having $\tilde{v}_{0}$ calculated above conditions $(1.10)_{2,3,4}$ are restrictions on $g_{0}$ and $h_{0}$.

In this paper we prove the existence of solutions to problem (1.1) in the HilbertSobolev-Slobodetski spaces $H^{2+s, 1+s / 2}\left(\Omega^{T}\right)$, where $s \in \mathbb{R}_{+} \cup\{0\}$. The proof is divided into two main steps: existence of solutions to problem (1.1) vanishing at time $t \leq 0$ (see Sect. 4) and appropriate extension of the initial data which transforms problem (1.1) to the problem (3.1) with vanishing initial data (see Sect. 5). The first step is much more difficult and crucial in the proof. It basis on local considerations (see Sect. 3) and some ideas of the regularizer (see [10, Ch. 4]).

The local considerations are connected with localization of problem (3.1) by an appropriate partition of unity (see Sect. 2). We distinguish two kinds of subdomains: interior and close to the boundary.

Then applying the partition of unity a solution to problem (3.1) is calculated in the form (4.1), where $v^{(k)}$ and $p^{(k)}$ are expressed explicitly (see Sect. 3). Problem (3.1) is considered in spaces $H_{\gamma}^{\sigma, \sigma / 2}, \gamma \geq 0, \sigma>0$ (see Definition 2.1), which are invariant with respect to an extension by zero for $t \leq 0$. The spaces are appropriate for examining the solvability of parabolic problems and were introduced by Agranovich and Vishik (see [1]) and were strongly developed by Solonnikov in [12] (see also [9, Vol. 2]).

The most natural way to find estimates and solve nonstationary parabolic problems with vanishing initial data is to find the Fourier-Laplace transforms of solutions and estimate them in spaces $\tilde{H}_{\gamma}^{\sigma, \sigma / 2}$ (see Definition 2.2). By Lemma 2.3 we have equivalence of norms of spaces $H_{\gamma}^{\sigma, \sigma / 2}$ and $\tilde{H}_{\gamma}^{\sigma, \sigma / 2}$ what expresses importance of spaces $H_{\gamma}^{\sigma, \sigma / 2}$.

The main goal of this paper is to prove the existence of solutions to problem (3.1) using only the existence of solutions to the heat equations in $\mathbb{R}^{3} \times \mathbb{R}, \mathbb{R}_{+}^{3} \times \mathbb{R}$ and to the Poisson equation in $\mathbb{R}^{3}, \mathbb{R}_{+}^{3}$. This can be made by applying the Fourier-Laplace transform and use estimates in spaces $\tilde{H}_{\gamma}^{\sigma, \sigma / 2}$ and $\tilde{H}_{\gamma}^{\sigma}$ respectively. This is possible because the Helmholtz-Weyl decomposition reduces solvability of (3.1) to solvability of the heat equation and the Poisson equation in the above mentioned spaces.

To show that our method is simple we recall the classical approach of solvability of an initial-boundary value problem for the nonstationary Stokes system (see [12,13,15, 16]). After appropriate transformation the nonstationary Stokes system with vanishing initial data in the half space $\mathbb{R}_{+}^{3} \times \mathbb{R}$, where $\mathbb{R}_{+}^{3}=\left\{x \in \mathbb{R}^{3}: x_{3}>0\right\}$ is derived.

Then after the Laplace transform with respect to $t$ and the Fourier transforms with respect to variables $x_{1}, x_{2}$ tangent to the plane $x_{3}=0$ a system of ordinary differential equations with respect to $x_{3}$ is formulated. Solving this system (see [12] for the Neumann boundary conditions and [7] for some parabolic system with slip boundary condition) and applying the definition of $\tilde{H}_{\gamma}^{\sigma, \sigma / 2}$ the appropriate estimate follows. 
In the $L_{p}$-approach we can distinguish two ways. First: solving the system in the half-space we construct a solution by using a corresponding Green function and next it is estimated by the Calderon-Zygmund theorem. Second: solving the ordinary differential system for the Fourier-Laplace transform we apply the MarcinkiewiczMikhlin theorem and obtain the estimate directly. The first approach was employed by Solonnikov in [13-16] in the case of non-slip and the Neumann boundary conditions. The second approach for the Neumann and the slip boundary conditions was used in $[2,3,11]$. In the fourth-coming paper we are going to extend the presented in this paper proof to the $L_{p}$-case.

\section{Notation and auxiliary results}

First we introduce the partition connected with a partition of unity $\left\{\omega^{(k)}, \Omega^{(k)}, \zeta^{(k)}(x)\right\}$, $k \in \mathcal{M} \cup \mathcal{N}$, such that $\zeta^{(k)}=\zeta^{(k)}(x)$ is a smooth function satisfying $0 \leq \zeta^{(k)}(x) \leq$ $1, \operatorname{supp} \zeta^{(k)}(x)=\Omega^{(k)}, \omega^{(k)}=\left\{x \in \Omega^{(k)}: \zeta^{(k)}(x)=1\right\}$.

Hence $\omega^{(k)} \subset \Omega^{(k)}$ and $\bigcup_{k \in \mathcal{M} \cup \mathcal{N}} \omega^{(k)}=\bigcup_{k \in \mathcal{M} \cup \mathcal{N}} \Omega^{(k)}=\Omega, \omega^{(k)}, \Omega^{(k)}$ for $k \in \mathcal{M}$ are interior subdomains but $\omega^{(k)}, \Omega^{(k)}, k \in \mathcal{N}$ are neighbourhoods near $S$. Moreover, we introduce functions $\vartheta^{(k)}(x)$ such that $\sum_{k \in \mathcal{M} \cup \mathcal{N}} \vartheta^{(k)}(x) \zeta^{(k)}(x)=1$.

We assume that at most $N_{0}$ of $\Omega^{(k)}$ has nonempty intersection, so

$$
\vartheta^{(k)}=\frac{\zeta^{(k)}}{\sum_{j}\left(\zeta^{(j)}\right)^{2}}
$$

Moreover, there exists $\lambda>0$ such that $\sup _{k} \operatorname{diam} \omega^{(k)} \leq \lambda, \sup _{k} \operatorname{diam} \Omega^{(k)} \leq$ $2 \lambda, \min _{k} \operatorname{dist}\left(\partial \Omega^{(k)}, \omega^{(k)}\right) \geq d \lambda, k \in \mathcal{M}, \min _{k} \operatorname{dist}\left(\left(\partial \Omega^{(k)} \backslash S\right),\left(\partial \omega^{(k)} \backslash S\right)\right) \geq d \lambda$, $k \in \mathcal{N}$ for some positive $d$. There is no relation between $N_{0}$ and $\lambda$. Hence we have

$$
\left|D_{x}^{\alpha} \zeta^{(k)}\right| \leq \frac{c}{\lambda^{|\alpha|}}, \quad\left|D_{x}^{\alpha} \vartheta^{(k)}\right| \leq \frac{c}{\lambda^{|\alpha|}}
$$

where $D_{x}^{\alpha}=\partial_{x_{1}}^{\alpha_{1}} \partial_{x_{2}}^{\alpha_{2}} \partial_{x_{3}}^{\alpha_{3}},|\alpha|=\alpha_{1}+\alpha_{2}+\alpha_{3}, \alpha_{i} \in \mathbb{N}_{0}=\mathbb{N} \cup\{0\}, \alpha=\left(\alpha_{1}, \alpha_{2}, \alpha_{3}\right)$ is a multiindex.

By $\xi^{(k)}$ we denote an interior point of $\omega^{(k)}$ and $\Omega^{(k)}$ for $k \in \mathcal{M}$ and the center of $\bar{\omega}^{(k)} \cap S$ and $\bar{\Omega}^{(k)} \cap S$ for $k \in \mathcal{N}$.

To consider problem (1.1) in a neighbourhood of $S$ we have to make it locally flat. Since problem (1.1) is invariant with respect to a translation and a rotation we can introduce a local system of coordinates with origin in $\xi^{(k)}, k \in \mathcal{N}$. We shall denote it by $y=\left(y_{1}, y_{2}, y_{3}\right)$ and $y=Y_{k}(x)$, where $Y_{k}$ is a composition of a translation and a rotation, which is such that the part $S^{(k)}=S \cap \bar{\Omega}^{(k)}$ of the boundary is described by $y_{3}=F_{k}\left(y_{1}, y_{2}\right), k \in \mathcal{N}$, where $F_{k} \in C^{s+2}$. Then we introduce new coordinates

$$
z_{i}=y_{i}, \quad i=1,2, \quad z_{3}=y_{3}-F_{k}\left(y_{1}, y_{2}\right)
$$


and denote the mapping by

$$
z=\Phi_{k}(y)
$$

Then we define

$$
\begin{aligned}
& \hat{\Omega}^{(k)}=\Phi_{k} \circ Y_{k}\left(\Omega^{(k)}\right) \equiv \Psi_{k}\left(\Omega^{(k)}\right), \\
& \hat{\omega}^{(k)}=\Phi_{k} \circ Y_{k}\left(\omega^{(k)}\right) \equiv \Psi_{k}\left(\omega^{(k)}\right), \quad k \in \mathcal{N} .
\end{aligned}
$$

We assume that the sets $\hat{\omega}^{(k)}, \hat{\Omega}^{(k)}$ are described in the local coordinates at $\xi^{(k)}$ by the inequalitites

$$
\begin{aligned}
& \left|y_{i}\right|<\lambda, \quad i=1,2, \quad 0<z_{3}=y_{3}-F_{k}\left(y_{1}, y_{2}\right)<\lambda, \\
& \left|y_{i}\right|<2 \lambda, \quad i=1,2, \quad 0<z_{3}=y_{3}-F_{k}\left(y_{1}, y_{2}\right)<2 \lambda
\end{aligned}
$$

Finally, we introduce the notation

$$
\begin{aligned}
& u^{(k)}(x, t)=u(x, t) \zeta^{(k)}(x, t), \\
& \hat{u}^{(k)}(z, t)=u\left(Y_{k}^{-1} \circ \Phi_{k}^{-1}(z), t\right), \\
& \tilde{u}^{(k)}(z, t)=\hat{u}^{(k)}(z, t) \hat{\zeta}^{(k)}(z)
\end{aligned}
$$

for $k \in \mathcal{N}$ and in the case of vector-valued function $u$ we have $\hat{u}_{i}^{(k)}(z, t)=0_{i j} u_{j}\left(Y_{k}^{-1}\right.$ o $\left.\Phi_{k}^{-1}(z), t\right)$, where $\left\{0_{i j}\right\}_{i, j=1,2,3}$ is an orthogonal matrix implied by the transformation $y_{i}=0_{i j} Y_{i}+r_{i}$ and $r_{i}$ describes translation. Moreover,

$$
u^{(k)}(x, t)=u(x, t) \zeta^{(k)}(x)
$$

for $k \in \mathcal{M}$.

Definition 2.1 We use the anisotropic Sobolev-Slobodetskii spaces $W_{2}^{l, l / 2}\left(Q^{T}\right), l \in$ $\mathbb{R}_{+}, Q^{T}=Q \times(0, T)$, where $Q$ is either $\Omega$ or $S$, with the norm (see $[1,7,12]$ )

$$
\begin{aligned}
\|u\|_{W_{2}^{l, l / 2}\left(Q^{T}\right)}^{2} & =\int_{0}^{T}\|u(t)\|_{W_{2}^{l}(Q)}^{2} d t+\int_{Q}\|u(x)\|_{W_{2}^{l / 2}(0, T)}^{2} d x \\
& \equiv\|u\|_{W_{2}^{l, 0}\left(Q^{T}\right)}^{2}+\|u\|_{W_{2}^{0, l / 2}\left(Q^{T}\right)}^{2},
\end{aligned}
$$

where

$$
\|u\|_{W_{2}^{l}(Q)}^{2}=\sum_{|\alpha| \leq l}\left\|D_{x}^{\alpha} u\right\|_{L_{2}(Q)}^{2} \equiv\|u\|_{W_{2}^{l-1}(Q)}^{2}+\|u\|_{W_{2}(Q)}^{2}
$$

for integer $l$, and 


$$
\begin{aligned}
\|u\|_{W_{2}^{l}(Q)}^{2}= & \sum_{|\alpha| \leq[l]}\left\|D_{x}^{\alpha} u\right\|_{L_{2}(Q)}^{2} \\
& +\sum_{|\alpha|=[l]} \int_{Q} \int_{Q} \frac{\left|D_{x}^{\alpha} u(x)-D_{x^{\prime}}^{\alpha} u\left(x^{\prime}\right)\right|^{2}}{\left|x-x^{\prime}\right|^{s+2(l-[l])}} d x d x^{\prime} \equiv\|u\|_{W_{2}^{[l]}(Q)}^{2}+\|u\|_{W_{2}(Q)}^{2}
\end{aligned}
$$

for noninteger $l$, where $s=\operatorname{dim} Q, D_{x}^{\alpha}=\partial_{x_{1}}^{\alpha_{1}} \ldots \partial_{x_{s}}^{\alpha_{s}}, \alpha=\left(\alpha_{1}, \ldots, \alpha_{s}\right)$ is a multiindes, $[l]$ is the integer part of $l$. For $Q=S$ the above norm is introduced by using local charts and a partition of unity.

Finally,

$$
\|u\|_{W_{2}^{l / 2}(0, T)}^{2}=\sum_{i \leq l / 2}\left\|\partial_{t}^{i} u\right\|_{L_{2}(0, T)}^{2}
$$

for integer $l / 2$, and

$$
\begin{aligned}
\|u\|_{W_{2}^{l / 2}(0, T)}^{2}= & \sum_{i \leq[l / 2]}\left\|\partial_{t}^{i} u\right\|_{L_{2}(0, T)}^{2} \\
& +\sum_{i=[l / 2]} \int_{0}^{T} \int_{0}^{T} \frac{\left|\partial_{t}^{i} u(t)-\partial_{t^{\prime}}^{i} u\left(t^{\prime}\right)\right|^{2}}{\left|t-t^{\prime}\right|^{1+2(l / 2-[l / 2])}} d t d t^{\prime},
\end{aligned}
$$

for $l / 2$ noninteger. We shall use the simplified notation

$$
H^{l, l / 2}\left(Q^{T}\right)=W_{2}^{l, l / 2}\left(Q^{T}\right) .
$$

To consider problems with vanishing initial conditions we need a space of functions which admits a zero extension to $t<0$. Therefore, for every $\gamma \geq 0$, we introduce the space $H_{\gamma}^{l, l / 2}\left(Q^{T}\right)$ with the norm (see $\left.[1,7,12]\right)$

$$
\|u\|_{H_{\gamma}^{l, l / 2}\left(Q^{T}\right)}^{2}=\int_{0}^{T} e^{-2 \gamma t}\|u\|_{W_{2}^{l}(Q)}^{2} d t+\|u\|_{H_{\gamma}^{0, l / 2}\left(Q^{T}\right)}^{2} .
$$

For $l / 2 \notin \mathbb{Z}$

$$
\begin{aligned}
& \|u\|_{H_{\gamma}^{0, l / 2}\left(Q^{T}\right)}^{2}=\gamma^{l} \int_{0}^{T} e^{-2 \gamma t}\|u\|_{L_{2}(Q)}^{2} d t \\
& +\int_{0}^{T} e^{-2 \gamma t} d t \int_{0}^{\infty} \frac{\left\|\partial_{t}^{k} u_{0}(\cdot, t-\tau)-\partial_{t}^{k} u_{0}(\cdot, t)\right\|_{L_{2}(Q)}^{2}}{\tau^{1+2(l / 2-k)}} d \tau,
\end{aligned}
$$


where $k=\left[\frac{l}{2}\right]<\frac{l}{2}$, and $u_{0}(x, t)=u(x, t)$ for $t>0, u_{0}(x, t)=0$ for $t<$ $0,\left.\partial_{t}^{k} u\right|_{t=0}=0$.

For $l / 2 \in \mathbb{Z}$,

$$
\|u\|_{H_{\gamma}^{0, l / 2}\left(Q^{T}\right)}^{2}=\int_{0}^{T} e^{-2 \gamma t}\left(\gamma^{l}\|u\|_{L_{2}(Q)}^{2}+\left\|\partial_{t}^{l / 2} u\right\|_{L_{2}(Q)}^{2}\right) d t
$$

and we assume that $\left.\partial_{t}^{j} u\right|_{t=0}=0, j=0, \ldots, l / 2-1$, so $u_{0}(x, t)$ has a generalized derivative $\partial_{t}^{l / 2} u_{0}$ in $Q \times(-\infty, T)$.

Functions in $H_{\gamma}^{l, l / 2}(Q \times(0, T))$ admit an extension by zero for $t<0$. A space of such elements we denote by $H_{\gamma}^{l, l / 2}(Q \times(-\infty, T))$.

In accordance with [1] we have that $u \in H_{\gamma}^{l, l / 2}(Q \times(0, T))$ if and only if

$$
u e^{-\gamma t} \in H^{l, l / 2}(Q \times(0, T)) \text { and } u_{\gamma} \in H^{l, l / 2}(Q \times(-\infty, T)), \quad T \leq 0,
$$

where

$$
u_{\gamma}= \begin{cases}e^{-\gamma t} u & \text { for } t>0 \\ 0 & \text { for } t<0\end{cases}
$$

Let us introduce the Fourier-Laplace transform for functions defined in $\mathbb{R}^{3} \times \mathbb{R}_{+}$by

$$
\tilde{u}(\xi, s)=\int_{\mathbb{R}^{3}} d x \int_{\mathbb{R}_{+}} e^{-s t+i \xi \cdot x} u(x, t) d t
$$

and for functions defined in $\mathbb{R}_{+}^{3} \times \mathbb{R}_{+}$by

$$
\tilde{u}\left(\xi, x_{3}, s\right)=\int_{\mathbb{R}^{2}} d x^{\prime} \int_{\mathbb{R}_{+}} e^{-s t+i \xi^{\prime} \cdot x^{\prime}} u\left(x^{\prime}, x_{3}, t\right) d t
$$

where $s=\gamma+i \xi_{0}, \operatorname{Res}=\gamma \geq 0, \xi_{0} \in \mathbb{R}, \xi \in \mathbb{R}^{3}, \xi \cdot x=\sum_{i=1}^{3} \xi_{i} x_{i}, \xi^{\prime} \cdot x^{\prime}=$ $\sum_{i=1}^{2} \xi_{i} x_{i}$.

For any function $u \in H_{\gamma}^{l, l / 2}\left(\mathbb{R}^{3} \times \mathbb{R}\right)$ the Laplace transform is defined for $\operatorname{Re} s \geq \gamma$. By the Paley-Wiener theorem the Laplace transform $\tilde{u}(x, s)$ is a holomorphic function of $s$ for $\operatorname{Re} s>\gamma$.

Definition 2.2 By $\tilde{H}_{\gamma}^{l, l / 2}\left(\mathbb{R}^{3} \times \mathbb{R}\right), l \in \mathbb{R}_{+}, \gamma \geq 0$, we define a space of functions with the finite norm

$$
\|u\|_{\tilde{H}_{\gamma}^{l, l / 2}\left(\mathbb{R}^{3} \times \mathbb{R}\right)}=\left(\int_{\mathbb{R}^{3}} d \xi \int_{\mathbb{R}} d \xi_{0}|\tilde{u}(\xi, s)|^{2}\left|s+\xi^{2}\right|^{l}\right)^{1 / 2},
$$

where $s=i \xi_{0}+\gamma$. 
Definition 2.3 By $\tilde{H}_{\gamma}^{l, l / 2}\left(\mathbb{R}_{+}^{3} \times \mathbb{R}\right), l \in \mathbb{R}_{+}, \gamma \geq 0$ we define a space of functions with the finite norm

$$
\begin{aligned}
& \|u\|_{\tilde{H}_{\gamma}^{l, l / 2}\left(\mathbb{R}_{+}^{3} \times \mathbb{R}\right)}=\left(\sum_{j \leq[l]} \int_{\mathbb{R}^{2}} d \xi^{\prime} \int_{\mathbb{R}} d \xi_{0} \int_{\mathbb{R}_{+}} d x_{3}\left|\partial_{x_{3}}^{j} \tilde{u}\left(\xi^{\prime}, x_{3}, s\right)\right|^{2}\left(\xi^{2}+|s|\right)^{l-j}\right. \\
& \left.\quad+\int_{\mathbb{R}^{2}} d \xi^{\prime} \int_{\mathbb{R}} d \xi_{0} \int_{R_{+}} \int_{\mathbb{R}_{+}} d x_{3}^{\prime} d x_{3}^{\prime \prime} \frac{\left|\partial_{x_{3}^{\prime}}^{[l]} \tilde{u}\left(\xi^{\prime}, x_{3}^{\prime}, s\right)-\partial_{x_{3}^{\prime \prime}}^{[l]} \tilde{u}\left(\xi^{\prime}, x_{3}^{\prime \prime}, s\right)\right|^{2}}{\left|x_{3}^{\prime}-x_{3}^{\prime \prime}\right|^{1+2(l-[l])}}\right)^{1 / 2},
\end{aligned}
$$

where $s=i \xi_{0}+\gamma$.

By the properties of the Parseval identity and Lemma 2.1 in [12] we have

Lemma 2.4 For any $\gamma \geq 0$ there exist constants $c_{1}$ and $c_{2}$ independent of $u$ and $\gamma, l$ such that

$$
c_{1}\|u\|_{H_{\gamma}^{l, l / 2}\left(\mathbb{R}^{3} \times \mathbb{R}\right)} \leq\|u\|_{\tilde{H}_{\gamma}^{l, l / 2}\left(\mathbb{R}^{3} \times \mathbb{R}\right)} \leq c_{2}\|u\|_{H_{\gamma}^{l, l / 2}\left(\mathbb{R}^{3} \times \mathbb{R}\right)} .
$$

Proof The proof is almost the same as the corresponding proof in [12, Sect. 2]. Since the result is very important we recall it for the reader convenience.

By the Fourier-Laplace transform (2.3) and the Parseval identity we have

$$
\int_{\mathbb{R}} d \xi_{0} \int_{\mathbb{R}^{3}}\left|\tilde{u}\left(\xi, \gamma+i \xi_{0}\right)\right|^{2} d \xi=(2 \pi)^{4} \int_{\mathbb{R}_{+}} e^{-2 \gamma t} d t \int_{\mathbb{R}^{3}}|u(x, t)|^{2} d x .
$$

The above identity implies

$$
\begin{aligned}
& \int_{\mathbb{R}_{+}} e^{-2 \gamma t}\|u\|_{\dot{W}_{2}^{l}\left(\mathbb{R}^{3}\right)}^{2} d t=c \int_{\mathbb{R}} \int_{\mathbb{R}^{3}} \sum_{|\alpha|=[l]}\left|\xi^{\alpha}\right|^{2}|\xi|^{2(l-[l])}|\tilde{u}|^{2} d \xi_{0} d \xi, \\
& \int_{\mathbb{R}_{+}} e^{-2 \gamma t}\|u\|_{L_{2}\left(\mathbb{R}^{3}\right)}^{2} \gamma^{l} d t=(2 \pi)^{-4} \int_{\mathbb{R}} \int_{\mathbb{R}^{3}} \gamma^{l}\left|\tilde{u}\left(\xi, \gamma+i \xi_{0}\right)\right|^{2} d \xi_{0} d \xi .
\end{aligned}
$$

Moreover, for noninteger $l / 2$ we have

$$
\begin{aligned}
& \int_{\mathbb{R}_{+}} e^{-2 \gamma t} d t \int_{\mathbb{R}_{+}}\left\|\frac{\partial^{k} u(\cdot, t-\tau)}{\partial t^{k}}-\frac{\partial^{k} u(\cdot, t)}{\partial t^{k}}\right\|_{L_{2}\left(\mathbb{R}^{3}\right)}^{2} \frac{d \tau}{\tau^{1+l-2 k}} \\
& =(2 \pi)^{-4} \int_{\mathbb{R}^{3}} d \xi \int_{\mathbb{R}^{2}}|s|^{2 k}|\tilde{u}(\xi, x)|^{2} d \xi_{0} \int_{0}^{\infty}\left|e^{-\tau s}-1\right|^{2} \frac{d \tau}{\tau^{1+l-2 k}},
\end{aligned}
$$

where $k=\left[\frac{l}{2}\right]$. 
Now we examine the last integral on the r.h.s. of (2.8). Changing variables $\tau^{\prime}=$ $|s| \tau,|s|=\sqrt{\gamma^{2}+\xi_{0}^{2}}$ we obtain

$$
\int_{0}^{\infty}\left|e^{-\tau s}-1\right|^{2} \frac{d \tau}{\tau^{1+l-2 k}}=|s|^{l-2 k} \int_{0}^{\infty}\left|e^{-\tau^{\prime} \frac{s}{|s|}}-1\right|^{2} \frac{d \tau^{\prime}}{\tau^{\prime} 1+l-2 k} \equiv|s|^{l-2 k} I(\infty) .
$$

Since

$$
\begin{aligned}
\mid e^{-\tau^{\prime} \frac{s}{|s|}} & -\left.1\right|^{2}=1+e^{-2 \tau^{\prime} \frac{\gamma}{|s|}}-2^{-\tau^{\prime} \frac{\gamma}{|s|}} \cos \frac{\xi_{0}}{|s|} \tau^{\prime} \\
= & \left(1-e^{-\tau^{\prime} \frac{\gamma}{|s|}}\right)^{2}+2 e^{-\tau^{\prime} \frac{\gamma}{|s|}}\left(1-\cos \frac{\xi_{0}}{|s|} \tau^{\prime}\right)
\end{aligned}
$$

behaves as $\tau^{\prime 2}$ near $\tau^{\prime}=0$ we obtain that $I(1)<\infty$, so there exist constants $c_{1}$ and $c_{2}$ such that

$$
c_{1} \leq I(\infty) \leq c_{2}
$$

because $l-2\left[\frac{l}{2}\right]>0$ for $\frac{l}{2}$ noninteger.

Hence

$$
\begin{aligned}
\tilde{c}_{1}\|u\|_{\tilde{H}_{\gamma}^{l, l / 2}\left(\mathbb{R}^{4}\right)}^{2} & \leq \int_{\mathbb{R}_{+}} e^{-2 \gamma t} d t \int_{\mathbb{R}_{+}}\left\|\frac{\partial^{k} u(\cdot, t-\tau)}{\partial t^{k}}-\frac{\partial^{k} u(\cdot, t)}{\partial t^{k}}\right\|_{L_{2}\left(\mathbb{R}^{3}\right)}^{2} \frac{d \tau}{\tau^{1+l-2 k}} \\
& \leq \tilde{c}_{2}\|u\|_{\tilde{H}_{\gamma}^{l, l / 2}\left(\mathbb{R}^{4}\right)}^{2}
\end{aligned}
$$

where $\tilde{c}_{1}, \tilde{c}_{2}$ do not depend on $\gamma$ and $u$.

For $\gamma=0$,

$$
\left|e^{-\tau^{\prime} i \frac{\xi_{0}}{\left|\xi_{0}\right|}}-1\right|^{2}=2-2 \cos \tau^{\prime} \frac{\xi_{0}}{\left|\xi_{0}\right|}
$$

Since the expression behaves as $\tau^{\prime 2}$ near $\tau^{\prime}=0$ the above considerations imply (2.9) for $\gamma=0$.

For $l / 2$ integer the above results also holds. This concludes the proof.

Remark 2.5 Similarly as Lemma 2.4 we prove. For any $\gamma \geq 0$ there exist positive constans $c_{1}$ and $c_{2}$ independent of $u$ and $\gamma$ such that

$$
c_{1}\|u\|_{H_{\gamma}^{l, l / 2}\left(\mathbb{R}_{+}^{3} \times \mathbb{R}\right)} \leq\|u\|_{\tilde{H}_{\gamma}^{l, l / 2}\left(\mathbb{R}_{+}^{3} \times \mathbb{R}\right)} \leq c_{2}\|u\|_{H_{\gamma}^{l, l / 2}\left(\mathbb{R}_{+}^{3} \times \mathbb{R}\right)} .
$$

We need the following interpolation inequality 
Lemma 2.6 (see $\left[12\right.$, Sect. 4]) Let $u \in H_{\gamma}^{l, l / 2}\left(\mathbb{R}_{T}^{3}\right), \gamma>0, l>0$. Let $\mathbb{R}_{T}^{3}=$ $\mathbb{R}^{3} \times(0, T)$. Let $\beta+|\alpha|+k<l$. Then

$$
\begin{aligned}
& \left\|\partial_{t}^{\beta / 2} D_{x}^{\alpha} u\right\|_{H_{\gamma}^{k, k / 2}\left(\mathbb{R}_{T}^{3}\right)} \leq \varepsilon^{l-|\alpha|-\beta-k}\|u\|_{H_{\gamma}^{l, l / 2}\left(\mathbb{R}_{T}^{3}\right)} \\
& \quad+c \varepsilon^{-k-|\alpha|-\beta}\left\|e^{-\gamma t} u\right\|_{L_{2}\left(\mathbb{R}_{T}^{3}\right)} \\
& \leq\left(\varepsilon^{l-|\alpha|-\beta-k}+c \gamma^{-l / 2} \varepsilon^{-k-|\alpha|-\beta}\right)\|u\|_{H_{\gamma}^{l, l / 2}\left(\mathbb{R}_{T}^{3}\right)} .
\end{aligned}
$$

Proof Inequality (2.11) is proved by Lemma 2.4 , the elementary inequality

$$
|s|^{\beta}|\xi|^{2|\alpha|}\left|s+\xi^{2}\right|^{k} \leq \varepsilon\left|s+\xi^{2}\right|^{l}+c(1 / \varepsilon)
$$

and the definition of space $H_{\gamma}^{l, l / 2}$.

Remark 2.7 Inequality (2.11) holds for $u \in H_{\gamma}^{l, l / 2}\left(\Omega^{T}\right)$ after applying a partition of unity in $\Omega$.

Lemma 2.8 Let us consider spaces $H_{\gamma}^{l, l / 2}(\Omega \times(-\infty, T)), l \in \mathbb{R}_{+}, \gamma \in[0, \infty)$. For any finite $T$ the norms of spaces $H_{\gamma_{1}}^{l, l / 2}(\Omega \times(-\infty, T))$ and $H_{\gamma_{2}}^{l, l / 2}(\Omega \times$ $(-\infty, T)), \gamma_{1}, \gamma_{2} \in[0, \infty)$ are equivalent.

The proof of the lemma follows directly from the definition of $H_{\gamma}^{l, l / 2}$ and the fact that elements of that space vanish for $t<0$.

Lemma 2.9 Let $b_{i} \in H^{2(l-i)+1}(\Omega), \Omega \subset \mathbb{R}^{n}, i \leq[l], l \in \mathbb{R}_{+}$. Then there exists $a$ function $u \in H^{2 l+2, l+1}\left(\Omega^{T}\right)$ such that

$$
\left.\partial_{t}^{i} u\right|_{t=0}=b_{i}, \quad i \leq[l]
$$

and

$$
\|u\|_{H^{2 l+2, l+1}\left(\Omega^{T}\right)} \leq c \sum_{i=0}^{[l]}\left\|b_{i}\right\|_{H^{2(l-i)+1}(\Omega)} .
$$

Lemma 2.10 Let $\mathbb{R}_{+}^{n}=\left\{x \in \mathbb{R}^{n}: x_{n}>0\right\}$. Let $a_{i} \in H^{l-i-1 / 2, l / 2-i / 2-1 / 4}\left(\mathbb{R}^{n-1} \times\right.$ $\left.\mathbb{R}_{+}\right), i \leq[l-1 / 2]$. Then there exists a function $u \in H^{l, l / 2}\left(\mathbb{R}_{+}^{n} \times \mathbb{R}_{+}\right)$such that

$$
\left.\partial_{x_{n}}^{i} u\right|_{x_{n}=0}=a_{i}, \quad i \leq[l-1 / 2]
$$

and the estimate holds

$$
\|u\|_{H^{l, l / 2}\left(\mathbb{R}_{+}^{n} \times \mathbb{R}_{+}\right)} \leq c \sum_{i=0}^{[l-1 / 2]}\left\|a_{i}\right\|_{H^{l-i-1 / 2, l / 2-i / 2-1 / 4}\left(\mathbb{R}^{n-1} \times \mathbb{R}_{+}\right)} .
$$


The anisotropic Sobolev-Slobodetski spaces $W_{p}^{l, l / 2}\left(\Omega^{T}\right), l \in \mathbb{R}_{+} \backslash \mathbb{Z}_{+}, p \in$ $(1, \infty)$ are called by Besov (see [4]) the generalized Sobolev spaces. They are equivalent to the Besov spaces $B_{p, p}^{l, l / 2}\left(\Omega^{T}\right)=B_{p}^{l, l / 2}\left(\Omega^{T}\right)$ (see also [4]). Then Lemmas 2.9 and 2.10 are special cases of Theorems 2.1 and 2.2 from [4]. For $l \in \mathbb{Z}$ Lemmas 2.9 and 2.10 follow from [5, Ch. 5]. Lemmas 2.9 and 2.10 for Besov spaces with mixed norms are proved in [6]. Theorems of direct and inverse traces for Hilbert-SobolevSlobodetski spaces were proved also in [9, Vol. 2, Ch. 4].

Finally we consider the problems

$$
\begin{array}{ll}
u_{t}-\Delta u=f & \text { in } R_{+}^{n} \times \mathbb{R}, \\
\left.u\right|_{x_{n}=0}=b_{1} & \text { on } \mathbb{R}^{n-1} \times \mathbb{R}
\end{array}
$$

and

$$
\begin{array}{ll}
u_{t}-\Delta u=f & \text { in } \mathbb{R}_{+}^{n} \times \mathbb{R}, \\
\left.\frac{\partial u}{\partial x_{n}}\right|_{x_{n}=0}=b_{2} & \text { on } \mathbb{R}^{n-1} \times \mathbb{R},
\end{array}
$$

where $x=\left(x_{1}, \ldots, x_{n}\right)=\left(x^{\prime}, x_{n}\right)$.

Following [7,12] we have

Lemma 2.11 Assume that

$$
\begin{aligned}
& f \in H_{\gamma}^{s, s / 2}\left(\mathbb{R}_{+}^{n} \times \mathbb{R}\right), \\
& b_{1} \in H_{\gamma}^{s+3 / 2, s / 2+3 / 4}\left(\mathbb{R}^{n-1} \times \mathbb{R}\right), \\
& b_{2} \in H_{\gamma}^{s+1 / 2, s / 2+1 / 4}\left(\mathbb{R}^{n-1} \times \mathbb{R}\right) .
\end{aligned}
$$

Then there exist solutions to problems (2.15) and (2.16) such that $u \in H_{\gamma}^{s+2, s / 2+1}\left(\mathbb{R}_{+}^{n} \times\right.$ $\mathbb{R})$ and the esitmates hold

$$
\begin{aligned}
\|u\|_{H_{\gamma}^{s+2, s / 2+1}\left(\mathbb{R}_{+}^{n} \times \mathbb{R}\right)} \leq c & \left(\|f\|_{H_{\gamma}^{s, s / 2}\left(\mathbb{R}_{+}^{n} \times \mathbb{R}\right)}\right. \\
& \left.+\left\|b_{1}\right\|_{H_{\gamma}^{s+3 / 2, s / 2+3 / 4}\left(\mathbb{R}^{n-1} \times \mathbb{R}\right)}\right)
\end{aligned}
$$

for (2.15), and

$$
\begin{aligned}
\|u\|_{H_{\gamma}^{s+2, s / 2+1}\left(\mathbb{R}_{+}^{n} \times \mathbb{R}\right)} \leq c & \left(\|f\|_{H_{\gamma}^{s, s / 2}\left(\mathbb{R}_{+}^{n} \times \mathbb{R}\right)}\right. \\
& \left.+\left\|b_{2}\right\|_{H_{\gamma}^{s+1 / 2, s / 2+1 / 4}\left(\mathbb{R}^{n-1} \times \mathbb{R}\right)}\right)
\end{aligned}
$$

for (2.16).

Proof Let us restrict our considerations to problem (2.15). Extending $f$ on $x_{n}<0$ by the Hestenes-Whitney method we solve the equation

$$
u_{t}-\Delta u=f^{\prime} \text { in } \mathbb{R}^{n} \times \mathbb{R}
$$


where $f^{\prime}$ is the extension of $f$, by applying the Fourier-Laplace transform (2.3) and using the Parseval identity to get solutions in spaces $H_{\gamma}^{s+2, s / 2+1}\left(\mathbb{R}^{n} \times \mathbb{R}\right)$ introduced in Definition 2.2.

Then (2.15) is transformed to the problem

$$
\begin{aligned}
& u_{t}-\Delta u=0, \\
& \left.u\right|_{x_{n}=0}=d .
\end{aligned}
$$

Applying transform (2.4) we get

$$
\tau^{2} \tilde{u}-\partial_{x_{n}}^{2} \tilde{u}=0,\left.\quad \tilde{u}\right|_{x_{n}=0}=\tilde{d}
$$

where $\tau=\sqrt{s+\left|\xi^{\prime}\right|^{2}}$. Solving (2.19) yields $\tilde{u}=\tilde{d} e^{-\tau x_{n}}$.

Using spaces $\tilde{H}_{\gamma}^{l, l / 2}\left(\mathbb{R}_{+}^{n} \times \mathbb{R}\right)$ introduced in Definition 2.3 and then Remark 2.5 (for details see $[7,12]$ ) we prove the lemma.

Let us consider the elliptic problem

$$
-\Delta u=f \text { in } \mathbb{R}^{3} \times \mathbb{R}
$$

where $t$ is parameter.

By the Fourier-Laplace transform and the Parseval identity we have

Lemma 2.12 Let $f \in H_{\gamma}^{s, s / 2+1}\left(\mathbb{R}^{3} \times \mathbb{R}\right), s \in \mathbb{R}_{+}, \gamma \geq 0$. Then for solutions to (2.20) we obtain

$$
\|u\|_{H_{\gamma}^{s+2, s / 2+1}\left(\mathbb{R}^{3} \times \mathbb{R}\right)} \leq c\|f\|_{H_{\gamma}^{s, s / 2+1}\left(\mathbb{R}^{3} \times \mathbb{R}\right)} .
$$

\section{Local considerations}

The aim of this section is to transform problem (1.1) with vanishing initial data to the Poisson equation in $\mathbb{R}^{3}, \mathbb{R}_{+}^{3}$ and the heat equation in $\mathbb{R}^{3} \times \mathbb{R}, \mathbb{R}_{+}^{3} \times \mathbb{R}$ for localized velocity and pressure. The localization is made by an appropriate partition of unity and the Helmholtz-Weyl decomposition.

Therefore we consider the following problem

$$
\begin{array}{ll}
v_{t}-v \Delta v+\nabla p=f & \text { in } \Omega \times \mathbb{R}, \\
\operatorname{div} v=g & \text { in } \Omega \times \mathbb{R}, \\
\bar{n} \cdot \mathbb{D}(v) \cdot \bar{\tau}_{\alpha}+\gamma v \cdot \bar{\tau}_{\alpha}=h_{\alpha}, \quad \alpha=1,2, & \text { on } S \times \mathbb{R}, \\
v \cdot \bar{n}=h_{3} & \text { on } S \times \mathbb{R}, \\
\int_{\Omega} p d x=0, &
\end{array}
$$

where we assumed that functions $f, g, h_{\alpha}, \alpha=1,2,3$, are extended by zero for $t<0$ and next by the Hestenes-Whitney method for $t>T$. 
First we consider problem (3.1) in an interior subdomain. Multiplying (3.1) by $\zeta^{(k)}, k \in \mathcal{M}$, and using notation (2.2) we obtain

$$
\begin{array}{ll}
v_{t}^{(k)}-v \Delta v^{(k)}+\nabla p^{(k)}=f_{1}^{(k)} & \text { in } \mathbb{R}^{3} \times \mathbb{R}, \\
\operatorname{div} v^{(k)}=g_{1}^{(k)} & \text { in } \mathbb{R}^{3} \times \mathbb{R},
\end{array}
$$

where

$$
\begin{aligned}
& f_{1}^{(k)}=-2 \nu \nabla \zeta^{(k)} \nabla v-v \Delta \zeta^{(k)} v+p \nabla \zeta^{(k)}+f^{(k)}, \\
& g_{1}^{(k)}=g^{(k)}+v \cdot \nabla \zeta^{(k)} .
\end{aligned}
$$

To apply the Helmholtz-Weyl decomposition we have to work with divergence free functions. For this purpose we introduce a function $\varphi^{(k)}$ by

$$
\Delta \varphi^{(k)}=g_{1}^{(k)} \text { in } \mathbb{R}^{3}
$$

Defining the new function

$$
u^{(k)}=v^{(k)}-\nabla \varphi^{(k)}
$$

we transform problem (3.2) into the following problem

$$
\begin{array}{ll}
u_{t}^{(k)}-v \Delta u^{(k)}+\nabla p^{(k)}=f_{1}^{(k)}-\nabla \varphi_{t}^{(k)}+v \Delta \nabla \varphi^{(k)} \equiv f_{2}^{(k)} & \text { in } \mathbb{R}^{3} \times \mathbb{R}, \\
\operatorname{div} u^{(k)}=0 & \text { in } \mathbb{R}^{3} \times \mathbb{R} .
\end{array}
$$

By the Helmholtz-Weyl decomposition (see [8, Vol. 1, Ch. 3]) there exist functions $\eta^{(k)}$ and $f_{3}^{(k)}$ such that

$$
f_{2}^{(k)}=\nabla \eta^{(k)}+f_{3}^{(k)}
$$

where $f_{3}^{(k)}$ is divergence free, so

$$
\operatorname{div} f_{3}^{(k)}=0
$$

and $\eta^{(k)}$ is a solution to the problem

$$
\Delta \eta^{(k)}=\operatorname{div} f_{2}^{(k)} \text { in } \mathbb{R}^{3} .
$$

Then problem (3.5) splits up into

$$
p^{(k)}=\eta^{(k)},
$$

where $\eta^{(k)}$ is a solution to (3.8) and

$$
u_{t}^{(k)}-v \Delta u^{(k)}=f_{3}^{(k)} \text { in } \mathbb{R}^{3} \times \mathbb{R} .
$$


Now we consider a neighbourhood near the boundary. Then for $k \in \mathcal{N}$ instead of (3.2) we have

$$
\begin{array}{lr}
v_{t}^{(k)}-v \Delta v^{(k)}+\nabla p^{(k)}=f_{1}^{(k)} & \text { in } \Omega^{(k)} \times \mathbb{R}, \\
\operatorname{div} v^{(k)}=g_{1}^{(k)} & \text { in } \Omega^{(k)} \times \mathbb{R}, \\
\nu \bar{n} \cdot \mathbb{D}\left(v^{(k)}\right) \cdot \bar{\tau}_{\alpha}+\gamma v^{(k)} \cdot \bar{\tau}_{\alpha}=h_{\alpha}^{(k)} & \\
\quad+v n_{i}\left(v_{j} \zeta_{, x_{i}}^{(k)}+v_{i} \zeta_{, x_{j}}^{(k)}\right) \tau_{\alpha j} \equiv h_{\alpha 1}^{(k)}, & \alpha=1,2, \\
v^{(k)} \cdot \bar{n}=h_{3}^{(k)} \equiv h_{31}^{(k)} & \text { on } S^{(k)} \times \mathbb{R},
\end{array}
$$

where $f_{1}^{(k)}$ and $g_{1}^{(k)}$ have the same form as in the case $k \in \mathcal{M}$ and the summation convention over the repeated indices is assumed.

Next we apply the mapping $\Psi_{k}$ and introduce the notation

$$
\begin{aligned}
& \nabla_{z}=\partial_{z}, \quad \nabla_{\Psi_{k j}}=\frac{\partial \Psi_{k i}}{\partial x_{j}} \cdot \nabla_{z_{i}}=\Psi_{k i, x_{j}} \cdot \nabla_{z_{i}}, \\
& \bar{n}_{z}=(0,0,1), \quad \bar{\tau}_{z 1}=(1,0,0), \quad \bar{\tau}_{z 2}=(0,1,0), \\
& \bar{n}_{\Psi_{k}}=\left.\frac{1}{\left|\nabla_{y} \chi_{k}\right|} \nabla_{y} \chi_{k}\right|_{y=\Psi_{k}^{-1}(z)}, \\
& \bar{\tau}_{\Psi_{k} 1}=\bar{n}_{\Psi_{k}} \times \bar{\tau}_{z 2}, \quad \bar{\tau}_{\Psi_{k} 2}=\bar{n}_{\Psi_{k}} \times \bar{\tau}_{z 1}
\end{aligned}
$$

and $\chi_{k}=y_{3}-F_{k}\left(y_{1}, y_{2}\right)$.

Applying mapping $\Psi_{k}$ and notation (2.1) problem (3.11) takes the form

$$
\begin{aligned}
& \tilde{v}_{t}^{(k)}-v \nabla_{z}^{2} \tilde{v}^{(k)}+\nabla_{z} \tilde{p}^{(k)} \\
& =-v\left(\nabla_{z}^{2} \tilde{v}^{(k)}-\nabla_{\Psi_{k}}^{2} \tilde{v}^{(k)}\right)+\nabla_{z} \tilde{p}^{(k)}-\nabla_{\Psi_{k}} \tilde{p}^{(k)} \\
& +\tilde{f}_{1}^{(k)} \equiv \tilde{f}_{2}^{(k)} \quad \text { in } \mathbb{R}_{+}^{3} \times \mathbb{R} \text {, } \\
& \operatorname{div}_{z} \tilde{v}^{(k)}=\operatorname{div}_{z} \tilde{v}^{(k)}-\operatorname{div}_{\Psi_{k}} \tilde{v}^{(k)}+\tilde{g}_{1}^{(k)} \equiv \tilde{g}_{2}^{(k)} \quad \text { in } \quad \mathbb{R}_{+}^{3} \times \mathbb{R}, \\
& \bar{n}_{z} \cdot \mathbb{D}_{z}\left(\tilde{v}^{(k)}\right) \cdot \bar{\tau}_{z \alpha}+\gamma \tilde{v}^{(k)} \cdot \bar{\tau}_{z \alpha} \\
& =\bar{n}_{z} \cdot \mathbb{D}_{z}\left(\tilde{v}^{(k)}\right) \cdot \bar{\tau}_{z \alpha}-\bar{n}_{\Psi_{k}} \cdot \mathbb{D}_{\Psi_{k}}\left(\tilde{v}^{(k)}\right) \cdot \bar{\tau}_{\Psi_{k} \alpha} \\
& +\gamma\left(\tilde{v}^{(k)} \cdot \bar{\tau}_{z \alpha}-\tilde{v}^{(k)} \cdot \bar{\tau}_{\Psi_{k} \alpha}\right)+\tilde{h}_{\alpha 1}^{(k)} \\
& \equiv \tilde{h}_{\alpha 2}^{(k)}, \quad \alpha=1,2, \quad z_{3}=0, \quad \text { on } \mathbb{R}^{2} \times \mathbb{R} \text {, } \\
& \bar{n}_{z} \cdot \tilde{v}^{(k)}=\bar{n}_{z} \cdot \tilde{v}^{(k)}-\bar{n}_{\Psi_{k}} \cdot \tilde{v}^{(k)}+\tilde{h}_{31}^{(k)} \equiv \tilde{h}_{32}^{(k)} z_{3}=0 \quad \text { on } \quad \mathbb{R}^{2} \times \mathbb{R} \text {. }
\end{aligned}
$$

Let us express problem (3.12) in the form

$$
\begin{array}{ll}
\tilde{v}_{t}^{(k)}-v \nabla_{z}^{2} \tilde{v}^{(k)}+\nabla_{z} \tilde{p}^{(k)}=\tilde{f}_{2}^{(k)} & z_{3}>0, \\
\operatorname{div}_{z} \tilde{v}^{(k)}=\tilde{g}_{2}^{(k)} & z_{3}>0, \\
\tilde{v}_{\alpha, z_{3}}^{(k)}=\tilde{h}_{\alpha 2}^{(k)}, \alpha=1,2, & z_{3}=0, \\
\tilde{v}_{3}^{(k)}=\tilde{h}_{32}^{(k)} & z_{3}=0 .
\end{array}
$$


Let $\varphi^{(k)}$ be a solution to the Neumann problem for the Poisson equation

$$
\begin{array}{ll}
\Delta \varphi^{(k)}=\tilde{g}_{2}^{(k)} & z_{3}>0 \\
\bar{n} \cdot \nabla \varphi^{(k)}=\tilde{h}_{32}^{(k)} & z_{3}=0 .
\end{array}
$$

Introducing the new function

$$
u^{(k)}=\tilde{v}^{(k)}-\nabla \varphi^{(k)}
$$

we see that $\left(u^{(k)}, \tilde{p}^{(k)}\right)$ is a solution to the problem

$$
\begin{array}{ll}
u_{t}^{(k)}-v \nabla_{z}^{2} u^{(k)}+\nabla_{z} \tilde{p}^{(k)}=\tilde{f}_{2}^{(k)}-\nabla \varphi_{t}^{(k)}+v \nabla \Delta \varphi^{(k)} \equiv \tilde{f}_{3}^{(k)}, & z_{3}>0 \\
\operatorname{div} u^{(k)}=0 & z_{3}>0 \\
u_{\alpha, z_{3}}^{(k)}=\tilde{h}_{\alpha 2}^{(k)}-\nabla_{z_{\alpha}} \varphi_{, z_{3}}^{(k)} \equiv \tilde{h}_{\alpha 3}^{(k)}, \quad \alpha=1,2, & z_{3}=0 \\
u_{3}^{(k)}=0 & z_{3}=0
\end{array}
$$

We need the Helmholtz-Weyl decomposition

$$
\tilde{f}_{3}^{(k)}=\nabla \eta^{(k)}+\tilde{f}_{4}^{(k)}, \quad \operatorname{div} \tilde{f}_{4}^{(k)}=0, \quad \tilde{f}_{4}^{(k)} \cdot \bar{n}_{z}=0 \quad \text { on } z_{3}=0 .
$$

Then

$$
\begin{array}{ll}
\Delta \eta^{(k)}=\operatorname{div} \tilde{f}_{3}^{(k)}, & z_{3}>0 \\
\bar{n}_{z} \cdot \nabla \eta^{(k)}=\bar{n}_{z} \cdot \tilde{f}_{3}^{(k)} & z_{3}=0 .
\end{array}
$$

Using the decomposition (3.17) in (3.16) yields

$$
\begin{array}{ll}
\tilde{p}^{(k)}=\eta^{(k)} & z_{3}>0 \\
\bar{n}_{z} \cdot \nabla \tilde{p}=\bar{n}_{z} \cdot \nabla \eta^{(k)}=\bar{n}_{z} \cdot \tilde{f}_{3}^{(k)} & z_{3}=0
\end{array}
$$

and $u^{(k)}$ is a solution to the problem

$$
\begin{array}{ll}
u_{t}^{(k)}-v \Delta_{z} u^{(k)}=\tilde{f}_{4}^{(k)} & z_{3}>0 \\
u_{\alpha, z_{3}}^{(k)}=\tilde{h}_{\alpha 3}^{(k)}, \quad \alpha=1,2, & z_{3}=0 \\
u_{3}^{(k)}=0 & z_{3}=0
\end{array}
$$

In view of the above considerations problem (3.1) is replaced by a system of local problems (3.8)-(3.10) for $k \in \mathcal{M}$ and (3.18)-(3.20) for $k \in \mathcal{N}$. Hence the complicated problem (3.1) is replaced by the Poisson and the heat equations in $\mathbb{R}^{3}$ and in $\mathbb{R}_{+}^{3}$, respectively. Solvability of these equations in the Sobolev-Slobodetski spaces $H_{\gamma}^{l, l / 2}$ can be shown by applying the Laplace-Fourier transform and the Parseval identity.

Since the Poisson Eqs. (3.8) and (3.18) and the heat Eqs. (3.10) and (3.20) determine local solutions (solutions with support in $\Omega^{(k)}, k \in \mathcal{M} \cup \mathcal{N}$ ) we describe solutions to (3.1) in $\Omega$ by using the properties of the partition of unity and express them in 
form (4.1). However, the above construction does not imply existence of solutions to problem (3.1) because functions $f_{2}^{(k)}, f_{3}^{(k)}$ for $k \in \mathcal{M}$ and $f_{3}^{(k)}, f_{4}^{(k)}$ for $k \in \mathcal{N}$ depend on $v$ and $p$.

Hence to prove the existence of solutions to (3.1) some fixed point argument must be applied. To make this possible the pressure $p$ occuring in the r.h.s. of Eqs. (3.8), (3.10), (3.18), (3.20) must be expressed in terms of $v$. This is possible for slip boundary conditions because in this case $p$ can be calculated from the Neumann problem to the Poisson equations (see [18]). The existence of solutions to (3.1) is proved in Sect. 4.

\section{Existence of solutions with vanishing initial data}

In this section we prove the existence of solutions to problem (3.1). To show this we use the local considerations from Sect. 3. By the property of the partition of unity we have

$$
\begin{aligned}
& v=\sum_{k \in \mathcal{M} \cup \mathcal{N}} \vartheta^{(k)} v^{(k)}, \\
& p=\sum_{k \in \mathcal{M} \cup \mathcal{N}} \vartheta^{(k)} p^{(k)} .
\end{aligned}
$$

Now we express $v^{(k)}$ and $p^{(k)}$ in the more explicit form. Let $k \in \mathcal{M}$. From (3.3), (3.4), (3.6), (3.7) and (3.10) we have

$$
v^{(k)}=u^{(k)}+\nabla \varphi^{(k)}
$$

where

$$
\begin{array}{r}
u^{(k)}-v \Delta u^{(k)}=f_{3}^{(k)} \text { in } \mathbb{R}^{3} \times \mathbb{R}, \\
f_{3}^{(k)}=f_{2}^{(k)}-\nabla \int_{\mathbb{R}^{3}} E(x-y) \operatorname{div}_{y} f_{2}^{(k)}(y, t) d y \\
f_{2}^{(k)}=f_{1}^{(k)}-\nabla \varphi_{t}^{(k)}+v \nabla \Delta \varphi^{(k)}, \\
f_{1}^{(k)}=-2 v \nabla \zeta^{(k)} \nabla v-v \Delta \zeta^{(k)} v+p \nabla \zeta^{(k)}+f^{(k)},
\end{array}
$$

where $E(x-y)$ is the fundamental solution to the Laplace equation and

$$
\Delta \varphi^{(k)}=g^{(k)}+v \cdot \nabla \zeta^{(k)} \text { in } \mathbb{R}^{3}
$$

Next

$$
p^{(k)}=\eta^{(k)}
$$


where

$$
\Delta \eta^{(k)}=\operatorname{div} f_{2}^{(k)} \text { in } \mathbb{R}^{3} .
$$

Hence

$$
\begin{aligned}
\left(v^{(k)}, p^{(k)}\right) & =L^{(k)}(v, p, f, g, h) \\
& \equiv\left(L_{1}^{(k)}(v, p, f, g, h), L_{2}^{(k)}(v, p, f, g, h)\right), \quad k \in \mathcal{M}
\end{aligned}
$$

where $L^{(k)}$ is an operator determined by relations (4.2)-(4.6).

Let $k \in \mathcal{N}$. Let $Z_{k}$ be such operator that

$$
Z_{k}: X\left(\hat{\Omega}^{(k)}\right) \rightarrow X\left(\Omega^{(k)}\right)
$$

for any function space $X$. Then

$$
v^{(k)}=Z_{k} \tilde{v}^{(k)}
$$

where $v^{(k)}$ and $\tilde{v}^{(k)}$ are solutions to problems (3.11) and (3.12), respectively. In view of (3.15) we have

$$
\tilde{v}^{(k)}=u^{(k)}+\nabla \varphi^{(k)}
$$

where $u^{(k)}$ is a solution to (3.16) and $\varphi^{(k)}$ is the function described by (3.14). Finally, we obtain

$$
\tilde{p}^{(k)}=\eta^{(k)}
$$

where $\eta^{(k)}$ is a solution to (3.18). Then

$$
p^{(k)}=Z_{k} \tilde{p}^{(k)}, \quad k \in \mathcal{N} .
$$

From (4.8)-(4.11) we obtain

$$
\left(v^{(k)}, p^{(k)}\right)=L^{(k)}(v, p, f, g, h) \text { for } k \in \mathcal{N} .
$$

Relations (4.1), (4.7), (4.12) imply

$$
(v, p)=\sum_{k \in \mathcal{M} \cup \mathcal{N}} \vartheta^{(k)} L^{(k)}(v, p, f, g, h) \equiv L(v, p, f, g, h)
$$

Existence of solutions to problem (3.1) is equivalent to find ( $v, p)$ satisfying (4.13). Let $\Phi(v, p)=L(v, p, f, g, h)$. Then solvability of (4.13) means an existence of a fixed point of the mapping

$$
(v, p)=\Phi(\hat{v}, \hat{p}) .
$$


For this we need to show that $\Phi$ is a contraction. This, however, is impossible if $p$ appears in (4.13). Hence to show the contraction we need a global estimate for pressure on $\Omega$. Let $\varphi$ be a solution to the Neumann problem

$$
\begin{array}{r}
\Delta \varphi=g \quad \text { in } \Omega, \\
\bar{n} \cdot \nabla \varphi=h_{3} \quad \text { on } S, \\
\int_{\Omega} \varphi d x=0,
\end{array}
$$

with the following compatibility condition

$$
\int_{\Omega} g d x=\int_{S} h_{3} d S .
$$

Introducing the new functions

$$
u=v-\nabla \varphi, \quad q=p+\varphi_{t}-v \Delta \varphi
$$

problem (3.1) takes the form

$$
\begin{aligned}
& u_{t}-v \Delta u+\nabla q=f, \\
& \operatorname{div} u=0, \\
& \left.u \cdot \bar{n}\right|_{S}=0, \\
& v \bar{n} \cdot \mathbb{D}(u) \cdot \bar{\tau}_{\alpha}+\gamma u \cdot \bar{\tau}_{\alpha}=h_{\alpha}-\nu \bar{n} \cdot \mathbb{D}(\nabla \varphi) \cdot \bar{\tau}_{\alpha}-\gamma \nabla \varphi \cdot \bar{\tau}_{\alpha} \\
& \quad \equiv d_{\alpha}, \quad \alpha=1,2 .
\end{aligned}
$$

Then $q$ is a solution to the problem

$$
\begin{array}{ll}
\Delta q=\operatorname{div} f & \text { in } \Omega, \\
\bar{n} \cdot \nabla q=f \cdot \bar{n}+v \bar{n} \cdot \Delta u & \text { on } S, \\
\int_{\Omega} q d x=-v \int_{S} h_{3} d S . &
\end{array}
$$

From [18] we have

$$
\left.\bar{n} \cdot \Delta u\right|_{S}=a_{\alpha \beta} u_{\alpha, \tau_{\beta}}+a_{\alpha} u_{\alpha}+b_{\alpha} u_{\alpha, n}-d_{\alpha, \tau_{\alpha}}
$$

where the summation convention with respect to $\alpha, \beta$ is assumed, $\alpha, \beta=1,2, \tau_{\alpha}, \alpha=$ 1,2 , are tangent coordinates to $S, n$ is the normal coordinate to $S, a_{\alpha \beta}, a_{\alpha}, b_{\alpha} \in C^{s}$ if $S \in C^{s+2}$. 
Lemma 4.1 Assume that $S \in C^{s+2}, h \in H_{\gamma}^{s+1 / 2, s / 2}(S \times \mathbb{R}), f \in H_{\gamma}^{s, s / 2}(\Omega \times$ $\mathbb{R}), \nabla \varphi \in H_{\gamma}^{s+2, s / 2+1}(\Omega \times \mathbb{R})$. Then the following inequality holds

$$
\begin{aligned}
& \|p\|_{H_{\gamma}^{s, s / 2}(\Omega \times \mathbb{R})}+\|\nabla p\|_{H_{\gamma}^{s, s / 2}(\Omega \times \mathbb{R})} \leq c\left(\|v\|_{H_{\gamma}^{s+1, s / 2}(\Omega \times \mathbb{R})}\right. \\
& +\|f\|_{H_{\gamma}^{s, s / 2}(\Omega \times \mathbb{R})}+\left\|\partial_{t} \nabla \varphi\right\|_{H_{\gamma}^{s, s / 2}(\Omega \times \mathbb{R})}+\left\|\nabla^{2} \nabla \varphi\right\|_{H_{\gamma}^{s, s / 2}(\Omega \times \mathbb{R})} \\
& \quad+\sum_{\alpha=1}^{2}\left(\left\|h_{\alpha}\right\|_{H^{s+1 / 2}\left(S ; L_{2, \gamma}(\mathbb{R})\right)}+\left\|h_{\alpha}\right\|_{H^{1 / 2}\left(S ; H_{\gamma}^{s / 2}(\mathbb{R})\right)}\right) \\
& \quad+\left\|h_{3}\right\|_{L_{2}\left(S ; H_{\gamma}^{s / 2}(\mathbb{R})\right)} .
\end{aligned}
$$

Proof Problem (4.20) is elliptic with respect to spatial variables where time $t$ is a parameter. Therefore to obtain an estimate for

$$
I=\|\nabla q\|_{H_{\gamma}^{s, s / 2}(\Omega \times \mathbb{R})}
$$

it is sufficient to obtain estimates for

$$
I_{1}=\|\nabla q\|_{L_{2}\left(\Omega ; H_{\gamma}^{s / 2}(\mathbb{R})\right)} \quad \text { and } \quad I_{2}=\|\nabla q\|_{L_{2, \gamma}\left(\mathbb{R} ; H^{s}(\Omega)\right)} .
$$

In view of the Laplace transform appeared in (2.3) and the definition of the space $\tilde{H}_{\gamma}^{s / 2}(\mathbb{R})$ with its equivalence to space $H_{\gamma}^{s / 2}(\mathbb{R})$ described by Lemma 2.3 we see that to estimate $I_{1}$ it is sufficient to find an estimate for $\|\nabla q\|_{L_{2}(\Omega)}$.

Multiplying (4.20) $)_{1}$ by $q$ and integrating over $\Omega$ we obtain

$$
\int_{S} \bar{n} \cdot \nabla q q d S-\int_{\Omega}|\nabla q|^{2} d x=\int_{S} \bar{n} \cdot f q d S-\int_{\Omega} f \cdot \nabla q d x .
$$

In view of (4.20) 2 and application of the Hölder and the Young inequalities to the last term on the r.h.s. we obtain the inequality

$$
\begin{aligned}
\|\nabla q\|_{L_{2}(\Omega)}^{2} & \leq c\|f\|_{L_{2}(\Omega)}^{2}+v \int_{S} \bar{n} \cdot \Delta u q d S \\
& \leq c\|f\|_{L_{2}(\Omega)}^{2}+\|\bar{n} \cdot \Delta u\|_{H^{-1 / 2}(S)}\|q\|_{H^{1 / 2}(S)} .
\end{aligned}
$$

In view of (4.21) we have

$$
\begin{aligned}
\|\bar{n} \cdot \Delta u\|_{H^{-1 / 2}(S)} & \leq c\|u\|_{H^{1}(\Omega)}+\sum_{\alpha=1}^{2}\left\|d_{\alpha}\right\|_{H^{1 / 2}(S)} \\
& \leq c\left(\|v\|_{H^{1}(\Omega)}+\|\nabla \varphi\|_{H^{2}(\Omega)}+\sum_{\alpha=1}^{2}\left\|h_{\alpha}\right\|_{H^{1 / 2}(S)}\right),
\end{aligned}
$$

where in the second inequality we used $(4.18)_{1}$ and (4.19) 4 . 
By the Poincare inequality and $(4.20)_{3}$ we have

$$
\|q\|_{L_{2}(\Omega)} \leq c\left(\|\nabla q\|_{L_{2}(\Omega)}+\left\|h_{3}\right\|_{L_{2}(S)}\right) .
$$

Using the above estimates in (4.23) yields

$$
\begin{aligned}
\|q\|_{H^{1}(\Omega)} \leq c & \left(\|v\|_{H^{1}(\Omega)}+\|\nabla \varphi\|_{H^{2}(\Omega)}+\|f\|_{L_{2}(\Omega)}\right. \\
& \left.+\sum_{\alpha=1}^{2}\left\|h_{\alpha}\right\|_{H^{1 / 2}(S)}+\left\|h_{3}\right\|_{L_{2}(S)}\right) .
\end{aligned}
$$

Applying the Laplace transform to (4.20) and (4.21), multiplying the results by $\mid \gamma+$ $\left.i \xi_{0}\right|^{s / 2}$, taking the inverse Laplace transform and repeating the considerations leading to (4.24) we obtain

$$
\begin{aligned}
& \|q\|_{H^{1}\left(\Omega ; H_{\gamma}^{s / 2}(\mathbb{R})\right)} \leq c\left(\|v\|_{H^{1}\left(\Omega ; H_{\gamma}^{s / 2}(\mathbb{R})\right)}\right. \\
& +\|\nabla \varphi\|_{H^{2}\left(\Omega ; H_{\gamma}^{s / 2}(\mathbb{R})\right)}+\|f\|_{L_{2}\left(\Omega ; H_{\gamma}^{s / 2}(\mathbb{R})\right)} \\
& \left.\quad+\sum_{\alpha=1}^{2}\left\|h_{\alpha}\right\|_{H^{1 / 2}\left(S ; H_{\gamma}^{s / 2}(\mathbb{R})\right)}+\left\|h_{3}\right\|_{L_{2}\left(S ; H_{\gamma}^{s / 2}(\mathbb{R})\right)}\right) .
\end{aligned}
$$

For solutions to (4.20) we have

$$
\begin{aligned}
& \|\nabla q\|_{H^{s}(\Omega)} \leq c\left(\|\operatorname{div} f\|_{H^{s-1}(\Omega)}+\|f \cdot \bar{n}+v \bar{n} \cdot \Delta u\|_{H^{s-1 / 2}(S)}\right) \\
& \leq c\left(\|f\|_{H^{s}(\Omega)}+\|u\|_{H^{s+1}(\Omega)}+\sum_{\alpha=1}^{2}\left\|d_{\alpha}\right\|_{H^{s+1 / 2}(S)}\right) \\
& \leq c\left(\|f\|_{H^{s}(\Omega)}+\|v\|_{H^{s+1}(\Omega)}+\left\|\nabla^{2} \varphi\right\|_{H^{s+1}(\Omega)}\right. \\
& \left.\quad+\sum_{\alpha=1}^{2}\left\|h_{\alpha}\right\|_{H^{s+1 / 2}(S)}\right)
\end{aligned}
$$

where we used (4.21) and (4.19) 4 .

Existence of solutions to problem (4.20) and estimate (4.26) follows from the theory developed in [9, Vol. 1]. The proof can be also made directly using [7,12]. Applying the partition of unity problem (4.20) is transformed to the local problems

$$
\Delta u=f \quad \text { in } \mathbb{R}^{3}
$$

and 


$$
\Delta u=0 \text { in } \mathbb{R}_{+}^{3}, \quad u=b \text { for } x_{3}=0 .
$$

Applying the Fourier transform with respect to $x^{\prime}$ to the problem in $\mathbb{R}_{+}^{3}$ we get

$$
-\left|\xi^{\prime}\right|^{2} \tilde{u}+\partial_{x_{3}}^{2} \tilde{u}=0,\left.\quad \tilde{u}\right|_{x_{3}=0}=\tilde{b}
$$

Solving we get $\tilde{u}=\tilde{b} e^{-\left|\xi^{\prime}\right| x_{3}}$. Using the definition of space $H^{l}\left(\mathbb{R}_{+}^{3}\right)$,

$$
\begin{aligned}
\|u\|_{H^{l}\left(\mathbb{R}_{+}^{3}\right)}= & \left(\sum_{j \leq[l]} \int_{\mathbb{R}^{2}} d \xi^{\prime} \int_{\mathbb{R}_{+}} d x_{3}\left|\partial_{x_{3}}^{j} \tilde{u}\right|^{2}\left|\xi^{\prime}\right|^{2(l-j)}\right. \\
& \left.+\int_{\mathbb{R}^{2}} d \xi^{\prime} \int_{\mathbb{R}_{+}} d x_{3}^{\prime} \int_{\mathbb{R}_{+}} d x_{3}^{\prime \prime} \frac{\left|\partial_{x_{3}^{\prime}}^{[l]} \tilde{u}\left(\xi^{\prime}, x_{3}^{\prime}\right)-\partial_{x_{3}^{\prime \prime}}^{[l]} \tilde{u}\left(\xi^{\prime}, x_{3}^{\prime \prime}\right)\right|^{2}}{\left|x_{3}^{\prime}-x_{3}^{\prime \prime}\right|^{1+2(l-[l]}}\right)^{1 / 2},
\end{aligned}
$$

the Parseval indentity, summing up local solutions and employing a perturbation argument we have existence of solutions to (4.20) and estimate (4.26).

From (4.25) and (4.26) we have

$$
\begin{aligned}
& \|\nabla q\|_{H_{\gamma}^{s, s / 2}(\Omega \times \mathbb{R})} \leq c\left(\|v\|_{H_{\gamma}^{s+1, s / 2}(\Omega \times \mathbb{R})}+\|f\|_{H_{\gamma}^{s, s / 2}(\Omega \times \mathbb{R})}\right. \\
& +\left\|\nabla \varphi_{t}\right\|_{H_{\gamma}^{s, s / 2}(\Omega \times \mathbb{R})}+\left\|\nabla^{2} \nabla \varphi\right\|_{H_{\gamma}^{s, s / 2}(\Omega \times \mathbb{R})} \\
& +\sum_{\alpha=1}^{2}\left(\left\|h_{\alpha}\right\|_{H^{s+1 / 2}\left(S ; L_{2, \gamma}(\mathbb{R})\right)}+\left\|h_{\alpha}\right\|_{H^{1 / 2}\left(S ; H_{\gamma}^{s / 2}(\mathbb{R})\right)}\right) \\
& \left.+\left\|h_{3}\right\|_{L_{2}\left(S ; H_{\gamma}^{s / 2}(\mathbb{R})\right)}\right) .
\end{aligned}
$$

From (4.18) 2 and (4.27) we obtain (4.22). This concludes the proof.

Lemma 4.2 Assume that $g \in H_{\gamma}^{s+1, s / 2}(\Omega \times \mathbb{R}), g_{t} \in H_{\gamma}^{s-1, s / 2}(\Omega \times \mathbb{R}), h_{3} \in$ $H_{\gamma}^{s+3 / 2,3 / 2}(S \times \mathbb{R}), h_{3 t} \in H_{\gamma}^{s-1 / 2, s / 2}(S \times \mathbb{R}), s \in \mathbb{R}_{+}$. Then for solutions to problem (4.15), (4.16) we have the estimates

$$
\|\nabla \varphi\|_{H_{\gamma}^{s+2, s / 2}(\Omega \times \mathbb{R})} \leq c\left(\|g\|_{H_{\gamma}^{s+1, s / 2}(\Omega \times \mathbb{R})}+\left\|h_{3}\right\|_{H_{\gamma}^{s+3 / 2, s / 2}(S \times \mathbb{R})}\right)
$$

and

$$
\left\|\partial_{t} \nabla \varphi\right\|_{H^{s, s / 2}(\Omega \times \mathbb{R})} \leq c\left(\left\|g_{t}\right\|_{H_{\gamma}^{s-1, s / 2}(\Omega \times \mathbb{R})}+\left\|h_{3 t}\right\|_{H_{\gamma}^{s-1 / 2, s / 2}(S \times \mathbb{R})}\right) .
$$

Moreover, we have also existence in the corresponding spaces. 
Proof Time dependence is not essential in proofs of (4.28) and (4.29) because (4.15), (4.16) is an elliptic problem and time is only a parameter. Since $s$ is real we prove the existence and estimates (4.28), (4.29) using [9, Ch. 2, Sect. 5.4]. The proofs can be made as follows. By a partition of unity we transform problem (4.15) to local problems in $\mathbb{R}^{3}$ and in $\mathbb{R}_{+}^{3}$. Then estimates in spaces $H^{s}, s \in \mathbb{R}_{+}$, follow by interpolation having estimates in $H^{k}, k \in \mathbb{N}$. For a bounded domain we can apply considerations from [9, Ch. 1, Sect. 7, 9]. This concludes the proof.

Remark 4.3 Proof of $I_{2}$ from the proof of Lemma 4.1 can be explained in the same way as it was made in the proof of Lemma 4.2.

Lemma 4.4 Let the assumptions of Lemmas 4.1, 4.2 be satisfied.

Then

$$
\begin{aligned}
& \|p\|_{H_{\gamma}^{s, s / 2}(\Omega \times \mathbb{R})}+\|\nabla p\|_{H_{\gamma}^{s, s / 2}(\Omega \times \mathbb{R})} \leq c\left(\|v\|_{H_{\gamma}^{s+1, s / 2}(\Omega \times \mathbb{R})}\right. \\
& \quad+\|f\|_{H_{\gamma}^{s, s / 2}(\Omega \times \mathbb{R})}+\left\|g_{t}\right\|_{H_{\gamma}^{s-1, s / 2}(\Omega \times \mathbb{R})}+\left\|h_{3, t}\right\|_{H_{\gamma}^{s-1 / 2, s / 2}(S \times \mathbb{R})} \\
& \quad+\|g\|_{H_{\gamma}^{s+1, s / 2}(\Omega \times \mathbb{R})}+\left\|h_{3}\right\|_{H_{\gamma}^{s+3 / 2, s / 2}(S \times \mathbb{R})} \\
& \left.\quad+\left\|h^{\prime}\right\|_{H^{s+1 / 2}\left(S ; L_{2, \gamma}(\mathbb{R})\right)}+\left\|h^{\prime}\right\|_{H^{1 / 2}\left(S ; H_{\gamma}^{s / 2}(\mathbb{R})\right)}\right) \\
& \equiv c\|v\|_{H_{\gamma}^{s+1, s / 2}(\Omega \times \mathbb{R})}+c F_{1}(s, f, g, h)
\end{aligned}
$$

where $h^{\prime}=\left(h_{1}, h_{2}\right)$.

In view of Lemma 4.4 we have that $p=\mathcal{F}(v)$, where $\mathcal{F}$ describes a linear functional with respect to $v$ such that

$$
\mathcal{F}: H_{\gamma}^{s+1, s / 2}(\Omega \times \mathbb{R}) \rightarrow H_{\gamma}^{s+1, s / 2}(\Omega \times \mathbb{R})
$$

and its estimate is expressed by (4.30).

Therefore, by (4.13), we obtain

$$
v=\sum_{k} \vartheta^{(k)} L_{1}^{(k)}(v, \mathcal{F}(v), f, g, h) \equiv L_{*}(v, f, g, h) .
$$

To show an existence of solutions to (4.31) we construct the mapping

$$
v=L_{*}(\tilde{v}, f, g, h) .
$$

We prove the existence of a fixed point to (4.32) by showing that $L_{*}(v, f, g, h)$ is a contraction with respect to $v$.

Lemma 4.5 Let the assumptions of Lemmas 4.1, 4.2 be satisfied.

Then $L_{*}(v, f, g, h)$ as the mapping with respect to $v$

$$
H_{\gamma}^{s+2, s / 2+1}(\Omega \times \mathbb{R}) \rightarrow H_{\gamma}^{s+2, s / 2+1}(\Omega \times \mathbb{R})
$$


is a contraction.

Proof We shall show that there exists $\delta \in(0,1)$ such that

$$
\begin{aligned}
& \left\|L_{*}(v, f, g, h)\right\|_{H_{\gamma}^{s+2, s / 2+1}(\Omega \times \mathbb{R})} \\
& \quad \leq \delta\|v\|_{H_{\gamma}^{s+2, s / 2+1}(\Omega \times \mathbb{R})}+c F(s, f, g, h)
\end{aligned}
$$

where

$$
\begin{aligned}
F(s, f, g, h)=\| & h\left\|_{H_{\gamma}^{s, s / 2}(\Omega \times \mathbb{R})}+\right\| g_{t} \|_{L_{2}\left(\Omega ; H_{\gamma}^{s / 2}(\mathbb{R})\right)} \\
& +\|g\|_{H_{\gamma}^{s+1, s / 2}(\Omega \times \mathbb{R})}+\left\|h^{\prime}\right\|_{H_{\gamma}^{s+1 / 2, s / 2+1 / 4}(S \times \mathbb{R})} \\
& +\left\|h_{3}\right\|_{H_{\gamma}^{s+3 / 2, s / 2+1}(S \times \mathbb{R})} .
\end{aligned}
$$

First we consider the case $k \in \mathcal{M}$. From (4.2) we have

$$
\begin{aligned}
& \left\|v^{(k)}\right\|_{H_{\gamma}^{s+2, s / 2+1}\left(\mathbb{R}^{3} \times \mathbb{R}\right)} \leq\left\|u^{(k)}\right\|_{H_{\gamma}^{s+2, s / 2+1}\left(\mathbb{R}^{3} \times \mathbb{R}\right)} \\
& +\left\|\nabla \varphi^{(k)}\right\|_{H_{\gamma}^{s+2, s / 2+1}\left(\mathbb{R}^{3} \times \mathbb{R}\right)} \equiv I_{1} .
\end{aligned}
$$

To estimate the first norm in $I_{1}$ we use problem (4.3). Since (4.3) is considered for all $x \in \mathbb{R}^{3}$ and all $t \in \mathbb{R}$ we can apply the Fourier-Laplace transform (2.3) and use spaces $\tilde{H}_{\gamma}^{l, l / 2}\left(\mathbb{R}^{3} \times \mathbb{R}\right)$ introduced in Definition 2.2. Then we have

$$
\left\|u^{(k)}\right\|_{\tilde{H}_{\gamma}^{s+2, s / 2+1}\left(\mathbb{R}^{3} \times \mathbb{R}\right)} \leq c\left\|f_{3}^{(k)}\right\|_{\tilde{H}_{\gamma}^{s, s / 2}\left(\mathbb{R}^{3} \times \mathbb{R}\right)} .
$$

Hence in view of $(4.3)_{2}$ we obtain

$$
\left\|u^{(k)}\right\|_{H_{\gamma}^{s+2, s / 2+1}\left(\mathbb{R}^{3} \times \mathbb{R}\right)} \leq c\left\|f_{2}^{(k)}\right\|_{H_{\gamma}^{s, s / 2}\left(\mathbb{R}^{3} \times \mathbb{R}\right)} .
$$

Now (4.37) and (4.3) 3 imply

$$
I_{1} \leq\left\|f_{1}^{(k)}\right\|_{H_{\gamma}^{s, s / 2}\left(\mathbb{R}^{3} \times \mathbb{R}\right)}+\left\|\nabla \varphi^{(k)}\right\|_{H_{\gamma}^{s+2, s / 2+1}\left(\mathbb{R}^{3} \times \mathbb{R}\right)}
$$

where

$$
\begin{array}{r}
\left\|f_{1}^{(k)}\right\|_{H_{\gamma}^{s, s / 2}\left(\mathbb{R}^{3} \times \mathbb{R}\right)} \leq c\left(\|v\|_{H_{\gamma}^{s+1, s / 2}\left(\mathbb{R}^{3} \cap \operatorname{supp} \zeta^{(k)} \times \mathbb{R}\right)}\right. \\
\left.+\|p\|_{H_{\gamma}^{s, s / 2}\left(\mathbb{R}^{3} \cap \operatorname{supp} \zeta^{(k)} \times \mathbb{R}\right)}+\left\|f^{(k)}\right\|_{H_{\gamma}^{s, s / 2}\left(\mathbb{R}^{3} \times \mathbb{R}\right)}\right)
\end{array}
$$


and

$$
\begin{aligned}
& \left\|\nabla \varphi^{(k)}\right\|_{H_{\gamma}^{s+2, s / 2+1}\left(\mathbb{R}^{3} \times \mathbb{R}\right)} \\
& =\left\|\nabla_{x} \int_{\mathbb{R}^{3}} E(x-y)\left(g_{t}^{(k)}+v \cdot \nabla \zeta^{(k)}\right)\right\|_{H_{\gamma}^{s+2, s / 2+1}\left(\mathbb{R}^{3} \times \mathbb{R}\right)} \\
& =\| \nabla_{x} \int_{\mathbb{R}^{3}} E(x-y)\left(g_{t}^{(k)}+v_{t} \cdot \nabla \zeta^{(k)} d y \|_{L_{2}\left(\mathbb{R}^{3} ; H_{\gamma}^{s / 2}(\mathbb{R})\right)} \equiv I_{2},\right. \\
& +\| \nabla_{x} \int_{\mathbb{R}^{3}} E(x-y)\left(g^{(k)}+v \cdot \nabla \zeta^{(k)} d y \|_{L_{2}\left(\mathbb{R} ; H_{\gamma}^{s+2}\left(\mathbb{R}^{3}\right)\right)} \equiv\right.
\end{aligned}
$$

where $E$ is the fundamental solution to the Laplace equation.

In view of Lemma 2.12 we have

$$
\begin{aligned}
I_{2} \leq c & \left(\left\|g_{t}^{(k)}\right\|_{L_{2}\left(\mathbb{R}^{3} ; H_{\gamma}^{s / 2}(\mathbb{R})\right)}+\left\|g^{(k)}\right\|_{H_{\gamma}^{s+1, s / 2}\left(\mathbb{R}^{3} \times \mathbb{R}\right)}\right. \\
& +\|v\|_{L_{2}\left(\mathbb{R} ; H_{\gamma}^{s+1}\left(\mathbb{R}^{3} \cap \operatorname{supp} \zeta^{(k)}\right)\right)} \\
& \left.+\left\|\nabla_{x} \int E(x-y) v_{t} \nabla \zeta^{(k)} d y\right\|_{L_{2}\left(\mathbb{R}^{3} ; H_{\gamma}^{s / 2}(\mathbb{R})\right)}\right) \equiv I_{3} .
\end{aligned}
$$

Using the Stokes system the last term in $I_{3}$ yields

$$
\begin{aligned}
& \left\|\nabla_{x} \int E(x-y)(v \Delta v-\nabla p+f) \nabla \zeta^{(k)} d y\right\|_{L_{2}\left(\mathbb{R}^{3} ; H_{\gamma}^{s / 2}(\mathbb{R})\right)} \\
& \leq c\left(\|v\|_{H^{1}\left(\mathbb{R}^{3} \cap \operatorname{supp} \zeta^{(k)} ; H^{s / 2}(\mathbb{R})\right)}+\|p\|_{L_{2}\left(\mathbb{R}^{3} \cap \operatorname{supp} \zeta^{(k)} ; H_{\gamma}^{s / 2}(\mathbb{R})\right)}\right. \\
& \left.\quad+\|f\|_{L_{2}\left(\mathbb{R}^{3} \cap \operatorname{supp} \zeta^{(k)} ; H_{\gamma}^{s / 2}(\mathbb{R})\right)}\right) .
\end{aligned}
$$

Summarizing the above considerations we have

$$
\begin{aligned}
& \left\|v^{(k)}\right\|_{H_{\gamma}^{s+2, s / 2+1}\left(\mathbb{R}^{3} \times \mathbb{R}\right)} \leq c\left(\|v\|_{H_{\gamma}^{s+1, s / 2}\left(\mathbb{R}^{3} \cap \operatorname{supp} \zeta^{(k)} \times \mathbb{R}\right)}\right. \\
& \quad+\|p\|_{H_{\gamma}^{s, s / 2}\left(\mathbb{R}^{3} \cap \operatorname{supp} \zeta^{(k)} \times \mathbb{R}\right)} \\
& \left.\quad+\|\nabla v\|_{L_{2}\left(\mathbb{R}^{3} \cap \operatorname{supp} \zeta^{(k)} ; H_{\gamma}^{s / 2}(\mathbb{R})\right.}\right)+c F_{1}^{(k)}(s),
\end{aligned}
$$

where

$$
\begin{aligned}
F_{1}^{(k)}(s)=\| & f^{(k)}\left\|_{H_{\gamma}^{s, s / 2}\left(\mathbb{R}^{3} \times \mathbb{R}\right)}+\right\| g_{t}^{(k)} \|_{L_{2}\left(\mathbb{R}^{3} ; H_{\gamma}^{s / 2}(\mathbb{R})\right)} \\
& +\left\|g^{(k)}\right\|_{H_{\gamma}^{s+1, s / 2}\left(\mathbb{R}^{3} \times \mathbb{R}\right)}+\|f\|_{L_{2}\left(\mathbb{R}^{3} \cap \operatorname{supp} \zeta^{(k)} ; H_{\gamma}^{s / 2}(\mathbb{R})\right)} .
\end{aligned}
$$


Let us consider the case $k \in \mathcal{N}$. Hence we want to estimate the norm

$$
\left\|v^{(k)}\right\|_{H_{\gamma}^{s+2, s / 2+1}\left(\Omega \cap \operatorname{supp} \zeta^{(k)} \times \mathbb{R}\right)} \cdot
$$

In view of the mapping $\Psi^{(k)}$ and formulas (2.1) we have

$$
\left\|v^{(k)}\right\|_{H_{\gamma}^{s+2, s / 2+1}\left(\Omega \cap \operatorname{supp}_{\zeta^{(k)}} \times \mathbb{R}\right)} \leq c\left\|\tilde{v}^{(k)}\right\|_{H_{\gamma}^{s+2, s / 2+1}\left(\mathbb{R}_{+}^{3} \times \mathbb{R}\right)} \equiv c I_{1},
$$

where functions $\tilde{v}^{(k)}$ and $\tilde{p}^{(k)}$ are solutions to problem (3.12).

In view of (3.15) we have

$$
I_{1} \leq\left\|\nabla \varphi^{(k)}\right\|_{H_{\gamma}^{s+2, s / 2+1}\left(\mathbb{R}_{+}^{3} \times \mathbb{R}\right)}+\left\|u^{(k)}\right\|_{H_{\gamma}^{s+2, s / 2+1}\left(\mathbb{R}_{+}^{3} \times \mathbb{R}\right)} \equiv I_{2} .
$$

Applying Lemma 2.11 to problem (3.20) yields

$$
\left\|u^{(k)}\right\|_{H_{\gamma}^{s+2, s / 2+1}\left(\mathbb{R}_{+}^{3} \times \mathbb{R}\right)} \leq c\left(\left\|\tilde{f}_{4}^{(k)}\right\|_{H_{\gamma}^{s, s / 2}\left(\mathbb{R}_{+}^{3} \times \mathbb{R}\right)}+\sum_{\alpha=1}^{2}\left\|\tilde{h}_{\alpha 3}^{(k)}\right\|_{H_{\gamma}^{s+1 / 2, s / 2+1 / 4}\left(\mathbb{R}^{2} \times \mathbb{R}\right)}\right) .
$$

From $(3.16)_{3}$ it follows

$$
\begin{aligned}
\sum_{\alpha=1}^{2}\left\|\tilde{h}_{\alpha 3}^{(k)}\right\|_{H_{\gamma}^{s+1 / 2, s / 2+1 / 4}\left(\mathbb{R}^{2} \times \mathbb{R}\right)} \leq & c \sum_{\alpha=1}^{2}\left\|\tilde{h}_{\alpha 2}^{(k)}\right\|_{H_{\gamma}^{s+1 / 2, s / 2+1 / 4}\left(\mathbb{R}^{2} \times \mathbb{R}\right)} \\
& +c\left\|\nabla \varphi^{(k)}\right\|_{H_{\gamma}^{s+2, s / 2+1}\left(\mathbb{R}_{+}^{3} \times \mathbb{R}\right)} .
\end{aligned}
$$

Continuing $(3.12)_{3}$ implies

$$
\begin{aligned}
& \sum_{\alpha=1}^{2}\left\|\tilde{h}_{\alpha 2}^{(k)}\right\|_{H_{\gamma}^{s+1 / 2, s / 2+1 / 4}\left(\mathbb{R}^{2} \times \mathbb{R}\right)} \leq c \lambda\left\|\tilde{v}^{(k)}\right\|_{H_{\gamma}^{s+2, s / 2+1}\left(\mathbb{R}_{+}^{3} \times \mathbb{R}\right)} \\
& \quad+c \sum_{\alpha=1}^{2}\left\|\tilde{h}_{\alpha 1}^{(k)}\right\|_{H_{\gamma}^{s+1 / 2, s / 2+1 / 4}\left(\mathbb{R}^{2} \times \mathbb{R}\right)}
\end{aligned}
$$

Finally in view of $(3.11)_{3}$ we obtain

$$
\begin{aligned}
& \sum_{\alpha=1}^{2}\left\|\tilde{h}_{\alpha 1}^{(k)}\right\|_{H_{\gamma}^{s+1 / 2, s / 2+1 / 4}\left(\mathbb{R}^{2} \times \mathbb{R}\right)} \leq c\left\|\hat{v}^{(k)}\right\|_{H_{\gamma}^{s+1, s / 2+1 / 2}\left(\mathbb{R}_{+}^{3} \times \mathbb{R}\right)} \\
& \quad+c \sum_{\alpha=1}^{2}\left\|h_{\alpha}^{(k)}\right\|_{H_{\gamma}^{s+1 / 2, s / 2+1 / 4}(S \times \mathbb{R})} .
\end{aligned}
$$


Now we shall estimate the first norm on the r.h.s. of (4.42). The decomposition (3.17) yields

$$
\left\|\tilde{f}_{4}^{(k)}\right\|_{H_{\gamma}^{s, s / 2}\left(\mathbb{R}_{+}^{3} \times \mathbb{R}\right)} \leq c\left(\left\|\tilde{f}_{3}^{(k)}\right\|_{H_{\gamma}^{s, s / 2}\left(\mathbb{R}_{+}^{3} \times \mathbb{R}\right)}+\left\|\nabla \eta^{(k)}\right\|_{H_{\gamma}^{s, s / 2}\left(\mathbb{R}^{3} \times \mathbb{R}\right)}\right)
$$

Solving problem (3.18) gives (see [9, Ch. 2, Sect. 5])

$$
\begin{aligned}
& \left\|\nabla \eta^{(k)}\right\|_{H_{\gamma}^{s, s / 2}\left(\mathbb{R}^{3} \times \mathbb{R}\right)} \leq c\left(\left\|\tilde{f}_{3}^{(k)}\right\|_{H_{\gamma}^{s, s / 2}\left(\mathbb{R}_{+}^{3} \times \mathbb{R}\right)}\right. \\
& \left.\quad+\left\|\bar{n}_{z} \cdot \tilde{f}_{3}^{(k)}\right\|_{H_{\gamma}^{s-1 / 2, s / 2-1 / 4}\left(\mathbb{R}^{2} \times \mathbb{R}\right)}\right) \leq c\left\|\tilde{f}_{3}^{(k)}\right\|_{H_{\gamma}^{s, s / 2}\left(\mathbb{R}_{+}^{3} \times \mathbb{R}\right)}
\end{aligned}
$$

where $s>1 / 2$.

Therefore, in view of (4.43) and (3.16) we obtain

$$
\begin{aligned}
& \left\|\tilde{f}_{4}^{(k)}\right\|_{H_{\gamma}^{s, s / 2}\left(\mathbb{R}_{+}^{3} \times \mathbb{R}\right)} \leq c\left\|\tilde{f}_{3}^{(k)}\right\|_{H_{\gamma}^{s, s / 2}\left(\mathbb{R}_{+}^{3} \times \mathbb{R}\right)} \\
& \quad \leq c\left(\left\|\tilde{f}_{2}^{(k)}\right\|_{H_{\gamma}^{s, s / 2}\left(\mathbb{R}_{+}^{3} \times \mathbb{R}\right)}+\left\|\nabla \varphi^{(k)}\right\|_{H_{\gamma}^{s+2, s / 2+1}\left(\mathbb{R}_{+}^{3} \times \mathbb{R}\right)}\right) .
\end{aligned}
$$

Next, (3.12) 1 implies

$$
\begin{aligned}
& \left\|\tilde{f}_{2}^{(k)}\right\|_{H_{\gamma}^{s, s / 2}\left(\mathbb{R}_{+}^{3} \times \mathbb{R}\right)} \leq c \lambda\left(\left\|\tilde{v}^{(k)}\right\|_{H_{\gamma}^{s+2, s / 2+1}\left(\mathbb{R}_{+}^{3} \times \mathbb{R}\right)}\right. \\
& \left.\quad+\left\|\nabla \tilde{p}^{(k)}\right\|_{H_{\gamma}^{s, s / 2}\left(\mathbb{R}_{+}^{3} \times \mathbb{R}\right)}\right)+c\left\|\tilde{f}_{1}^{(k)}\right\|_{H_{\gamma}^{s, s / 2}\left(\mathbb{R}_{+}^{3} \times \mathbb{R}\right)}
\end{aligned}
$$

Finally,

$$
\begin{aligned}
& \left\|\tilde{f}_{1}^{(k)}\right\|_{H_{\gamma}^{s, s / 2}\left(\mathbb{R}_{+}^{3} \times \mathbb{R}\right)} \leq c\|\hat{v}\|_{H_{\gamma}^{s+1, s / 2}\left(\mathbb{R}_{+}^{3} \cap \operatorname{supp} \hat{\zeta}^{(k)} \times \mathbb{R}\right)} \\
& \quad+c\|\hat{p}\|_{H_{\gamma}^{s, s / 2}\left(\mathbb{R}_{+} \cap \operatorname{supp} \hat{\zeta}^{(k)} \times \mathbb{R}\right)}+c\left\|f^{(k)}\right\|_{H_{\gamma}^{s, s / 2}(\Omega \times \mathbb{R})} .
\end{aligned}
$$

Using the above estimates in $I_{2}$ yields

$$
\begin{aligned}
I_{2} \leq c & \left(\left\|\tilde{v}^{(k)}\right\|_{H_{\gamma}^{s+2, s / 2+1}\left(\mathbb{R}_{+}^{3} \times \mathbb{R}\right)}+\left\|\nabla \tilde{p}^{(k)}\right\|_{H_{\gamma}^{s, s / 2}\left(\mathbb{R}_{+}^{3} \times \mathbb{R}\right)}\right) \\
& +c\left(\|\hat{v}\|_{H_{\gamma}^{s+1, s / 2+1 / 2}\left(\mathbb{R}_{+}^{3} \cap \operatorname{supp} \hat{\zeta}^{(k)} \times \mathbb{R}\right)}\right. \\
& \left.+\|\hat{p}\|_{H_{\gamma}^{s, s / 2}\left(\mathbb{R}_{+}^{3} \cap \operatorname{supp} \hat{\zeta}^{(k)} \times \mathbb{R}\right)}\right)+c \sum_{\alpha=1}^{2}\left\|h_{\alpha}^{(k)}\right\|_{H_{\gamma}^{s+1 / 2, s / 2+1 / 4}(\Omega \times \mathbb{R})} \\
& +c\left\|f^{(k)}\right\|_{H_{\gamma}^{s, s / 2}(\Omega \times \mathbb{R})}+c\left\|\nabla \varphi^{(k)}\right\|_{H_{\gamma}^{s+2, s / 2+1}\left(\mathbb{R}_{+}^{3} \times \mathbb{R}\right)} \\
\equiv & I_{3} .
\end{aligned}
$$


Now we estimate the last norm in $I_{3}$. Using that $G$ is the Green function to the Neumann problem (3.14) we have

$$
\begin{aligned}
& \left\|\nabla \varphi^{(k)}\right\|_{H_{\gamma}^{s+2, s / 2+1}\left(\mathbb{R}_{+}^{3} \times \mathbb{R}\right)}=\left\|\nabla_{z} \int_{\mathbb{R}_{+}^{3}} G(z, y) \tilde{g}_{2}^{(k)} d y\right\|_{H_{\gamma}^{s+2, s / 2+1}\left(\mathbb{R}_{+}^{3} \times \mathbb{R}\right)} \\
& +\left\|\nabla_{z} \int_{\mathbb{R}^{2}} G\left(z, y^{\prime}\right) \tilde{h}_{32}^{(k)} d y^{\prime}\right\|_{H_{\gamma}^{s+2, s / 2+1}\left(\mathbb{R}_{+}^{3} \times \mathbb{R}\right)} \equiv J_{1}+J_{2} .
\end{aligned}
$$

First we consider $J_{1}$. Using the form of $\tilde{g}_{2}^{(k)}$ from (3.12) 2 and $\tilde{g}_{1}^{(k)}$ from (3.2) we have

$$
\begin{aligned}
J_{1}=\| & \nabla_{y} \int_{\mathbb{R}_{+}^{3}} G(y, z)\left[\operatorname{div}_{z} \tilde{v}^{(k)}-\operatorname{div} \Psi_{k} \tilde{v}^{(k)}+\hat{v} \cdot \nabla_{\Psi_{k}} \hat{\zeta}^{(k)}\right. \\
& \left.\quad+\hat{g}^{(k)}\right] d z \|_{H_{\gamma}^{s+2, s / 2+1}\left(\mathbb{R}_{+}^{3} \times \mathbb{R}\right)} \\
\leq & c \lambda\left\|\tilde{v}^{(k)}\right\|_{H_{\gamma}^{s+2, s / 2+1}\left(\mathbb{R}_{+}^{3} \times \mathbb{R}\right)}+c\left\|\hat{g}^{(k)}\right\|_{H_{\gamma}^{s+1, s / 2+1}\left(\mathbb{R}_{+}^{3} \times \mathbb{R}\right)} \\
& +\left\|\nabla_{y} \int_{\mathbb{R}_{+}^{3}} G(y, z) \hat{v} \nabla_{\Psi_{k}} \hat{\zeta}^{(k)} d z\right\|_{H_{\gamma}^{s+2, s / 2+1}\left(\mathbb{R}_{+}^{3} \times \mathbb{R}\right)} .
\end{aligned}
$$

The last norm in the above inequality we split as follows

$$
\begin{aligned}
& \left\|\nabla_{y} \int_{\mathbb{R}_{+}^{3}} G(y, z) \hat{v} \cdot \nabla_{\Psi_{k}} \hat{\zeta}^{(k)} d z\right\|_{L_{2, \gamma}\left(\mathbb{R} ; H^{s+2}\left(\mathbb{R}_{+}^{3}\right)\right.} \\
& +\left\|\nabla_{y} \int_{\mathbb{R}_{+}^{3}} G(y, z) \hat{v} \cdot \nabla_{\Psi_{k}} \hat{\zeta}^{(k)} d z\right\|_{L_{2}\left(\mathbb{R}_{+}^{3} ; H_{\gamma}^{s / 2+1}(\mathbb{R})\right)} \equiv K_{1}+K_{2},
\end{aligned}
$$

where

$$
K_{1} \leq c\|\hat{v}\|_{L_{2, \gamma}\left(\mathbb{R} ; H^{s+1}\left(\mathbb{R}_{+}^{3} \cap \operatorname{supp} \hat{\zeta}^{(k)}\right)\right)}
$$

and

$$
K_{2}=\left\|\nabla_{y} \int_{\mathbb{R}_{+}^{3}} G(y, z) \hat{v}_{t} \nabla_{\Psi_{k}} \hat{\zeta}^{(k)} d z\right\|_{L_{2}\left(\mathbb{R}_{+}^{3} ; H_{\gamma}^{s / 2}(\mathbb{R})\right)}
$$


Using Eq. (3.1) 1 transformed to variables $z$ we get

$$
\begin{aligned}
& K_{2}=\left\|\nabla_{y} \int_{\mathbb{R}_{+}^{3}} G(y, z)\left(\operatorname{div} \Psi_{k} \mathbb{T}_{\Psi_{k}}\left(\hat{v}^{(k)}, \hat{p}^{(k)}\right)+\hat{f}^{(k)}\right) \hat{\nabla}_{\Psi_{k}} \hat{\zeta}^{(k)}\right\|_{L_{2}\left(\mathbb{R}_{+}^{3} ; H_{\gamma}^{s / 2}(\mathbb{R})\right)} \\
& \leq c\left(\left\|\hat{v}^{(k)}\right\|_{L_{2}\left(\mathbb{R}_{+}^{3} ; H_{\gamma}^{s / 2}(\mathbb{R})\right)}+\left\|\hat{p}^{(k)}\right\|_{L_{2}\left(\mathbb{R}_{+}^{3} ; H_{\gamma}^{s / 2}(\mathbb{R})\right)}\right. \\
& \left.\quad+\left\|\hat{f}^{(k)}\right\|_{L_{2}\left(\mathbb{R}_{+}^{3} ; H_{\gamma}^{s / 2}(\mathbb{R})\right)}\right),
\end{aligned}
$$

where we use the Poincare inequality to have the estimate

$$
\left\|\hat{p}^{(k)}\right\|_{L_{2}\left(\mathbb{R}_{+}^{3} ; H_{\gamma}^{s / 2}(\mathbb{R})\right)} \leq c \lambda\left\|\nabla \hat{p}^{(k)}\right\|_{L_{2}\left(\mathbb{R}_{+}^{3} ; H_{\gamma}^{s / 2}(\mathbb{R})\right)} .
$$

Finally,

$$
J_{2} \leq c \lambda\left\|\tilde{v}^{(k)}\right\|_{H_{\gamma}^{s+2, s / 2+1}\left(\mathbb{R}_{+}^{3} \times \mathbb{R}\right)}+c\left\|\tilde{h}_{3}^{(k)}\right\|_{H_{\gamma}^{s+3 / 2, s / 2+1}\left(\mathbb{R}^{2} \times \mathbb{R}\right)}
$$

Summarizing the above estimates we obtain

$$
\begin{aligned}
& \left\|v^{(k)}\right\|_{H_{\gamma}^{s+2, s / 2+1}(\Omega \times \mathbb{R})} \leq c \lambda\left(\left\|\tilde{v}^{(k)}\right\|_{H_{\gamma}^{s+2, s / 2+1}\left(\mathbb{R}_{+}^{3} \times \mathbb{R}\right)}\right. \\
& \left.+\left\|\nabla \tilde{p}^{(k)}\right\|_{H_{\gamma}^{s, s / 2}\left(\mathbb{R}_{+}^{3} \times \mathbb{R}\right)}\right)+c\left(\|v\|_{H_{\gamma}^{s+1, s / 2+1 / 2}\left(\Omega \cap \operatorname{supp} \zeta^{(k)} \times \mathbb{R}\right)}\right. \\
& \left.+\|p\|_{H_{\gamma}^{s, s / 2}\left(\Omega \cap \operatorname{supp} \zeta^{(k)} \times \mathbb{R}\right)}\right)+c\left(\left\|f^{(k)}\right\|_{H_{\gamma}^{s, s / 2}(\Omega \times \mathbb{R})}\right. \\
& \quad+\left\|\tilde{g}_{t}^{(k)}\right\|_{L_{2}\left(\mathbb{R}_{+}^{3} ; H_{\gamma}^{s / 2}(\mathbb{R})\right)}+\left\|\tilde{g}^{(k)}\right\|_{L_{2, \gamma}\left(\mathbb{R} ; H^{s+1}\left(\mathbb{R}_{+}^{3}\right)\right)} \\
& \left.\quad+\sum_{\alpha=1}^{2}\left\|\tilde{h}_{\alpha}^{(k)}\right\|_{H_{\gamma}^{s+1 / 2, s / 2+1 / 4}\left(\mathbb{R}^{2} \times \mathbb{R}\right)}+\left\|\tilde{h}_{3}^{(k)}\right\|_{H_{\gamma}^{s+3 / 2, s / 2+1}\left(\mathbb{R}^{2} \times \mathbb{R}\right)}\right) .
\end{aligned}
$$

Adding (4.38) and (4.44) for all $k \in \mathcal{M} \cup \mathcal{N}$, passing to the old variables $x$ and using (4.30) yields

$$
\begin{aligned}
& \|v\|_{H_{\gamma}^{s+2, s / 2+1}(\Omega \times \mathbb{R})} \\
& \leq c\left(\lambda+\varepsilon+c(1 / \varepsilon) \gamma^{-1}\right)\|v\|_{H_{\gamma}^{s+2, s / 2+1}(\Omega \times \mathbb{R})}+c F(s, f, g, h),
\end{aligned}
$$

where $F(s, f, g, h)$ is defined by (4.34). For $\lambda, \varepsilon$ sufficiently small and $\gamma$ sufficiently large we obtain (4.33). This concludes the proof.

Now we shall formulate the main result of this section

Theorem 4.6 Assume that $s \in \mathbb{R}_{+}$. Assume that $f, g$, $h$ are such that quantity (4.34) is finite. Assume that $S \in C^{s+2}$ and $\gamma>0$ is sufficiently large. Then there exists a 
solution to problem (3.1) such $v \in H_{\gamma}^{s+2, s / 2+1}(\Omega \times \mathbb{R}), \nabla p \in H_{\gamma}^{s, s / 2}(\Omega \times \mathbb{R})$ and

$$
\|v\|_{H_{\gamma}^{s+2, s / 2+1}(\Omega \times \mathbb{R})}+\|\nabla p\|_{H_{\gamma}^{s, s / 2}(\Omega \times \mathbb{R})} \leq c F(s, f, g, h) .
$$

\section{Proof of Theorem A}

The proof is based on a transformation of problem (1.1) to problem (3.1) with vanishing initial data. Let us consider problem (1.1). Let $\tilde{v}_{0} \in H^{s+2, s / 2+1}\left(\Omega^{T}\right)$ be an extension of the initial data such that (see Lemma 2.9)

$$
\left\|\tilde{v}_{0}\right\|_{H^{s+2, s / 2+1}\left(\Omega^{T}\right)} \leq c\left\|v_{0}\right\|_{H^{s+1}(\Omega)}
$$

and

$$
\left.\tilde{v}_{0}\right|_{t=0}=v_{0}
$$

Introducing the new function

$$
u=v-\tilde{v}_{0}
$$

we see that $u$ and $p$ are solutions to the problem

$$
\begin{array}{ll}
u_{t}-\operatorname{div} \mathbb{T}(u, p)=f_{0}-\tilde{v}_{0 t}+v \operatorname{div} \mathbb{D}\left(\tilde{v}_{0}\right) \equiv f & \text { in } \Omega^{T}, \\
\operatorname{div} u=g_{0}-\operatorname{div} \tilde{v}_{0} \equiv g & \text { in } \Omega^{T}, \\
\nu \bar{n} \cdot \mathbb{D}(u) \cdot \bar{\tau}_{\alpha}+\gamma u \cdot \bar{\tau}_{\alpha}=h_{0 \alpha}-v \bar{n} \cdot \mathbb{D}\left(\tilde{v}_{0}\right) \cdot \bar{\tau}_{\alpha} & \\
\quad-\gamma \tilde{v}_{0} \cdot \bar{\tau}_{\alpha} \equiv h_{\alpha}, \alpha=1,2, & \text { on } S^{T}, \\
u \cdot \bar{n}=h_{03}-\tilde{v}_{0} \cdot \bar{n} \equiv h_{3} & \text { on } S^{T}, \\
\left.u\right|_{t=0}=0 & \text { in } \Omega .
\end{array}
$$

By the compatibility conditions (1.10) we obtain that

$$
\begin{aligned}
& f \in H_{0}^{s, s / 2}\left(\Omega^{T}\right), \quad g \in H_{0}^{s+1, s / 2}\left(\Omega^{T}\right), \quad g_{t} \in H_{0}^{s, s / 2}\left(\Omega^{T}\right), \\
& h^{\prime}=\left(h_{1}, h_{2}\right) \in H_{0}^{s+1 / 2, s / 2+1 / 4}\left(S^{T}\right), \quad h_{3} \in H_{0}^{s+3 / 2, s / 2+3 / 4}\left(S^{T}\right) .
\end{aligned}
$$

By the properties of spaces $H_{0}^{l, l / 2}$ we know that functions $f, g, h$ can be extended by zero for $t<0$ and the extended functions belong to the same norms.

For $T<\infty$ the norms $H_{\gamma}^{l, l / 2}\left(Q^{T}\right)$ and $H_{0}^{l, l / 2}\left(Q^{T}\right)$ are equivalent (see Lemma 2.8). Hence for $T<\infty$,

$$
\begin{aligned}
& \|f\|_{H_{\gamma}^{s, s / 2}\left(\Omega^{T}\right)} \leq c(T)\|f\|_{H_{0}^{s, s / 2}\left(\Omega^{T}\right)}, \\
& \|g\|_{H_{\gamma}^{s+1, s}\left(\Omega^{T}\right)} \leq c(T)\|g\|_{H_{0}^{s+1, s}\left(\Omega^{T}\right)}, \\
& \left\|h^{\prime}\right\|_{H_{\gamma}^{s+1 / 2, s / 2+1 / 4}\left(S^{T}\right)} \leq c(T)\left\|h^{\prime}\right\|_{H_{0}^{s+1 / 2, s / 2+1 / 4}\left(S^{T}\right)}, \\
& \left\|h_{3}\right\|_{H_{\gamma}^{s+3 / 2, s / 2+3 / 4}\left(S^{T}\right)} \leq c(T)\left\|h_{3}\right\|_{H_{0}^{s+3 / 2, s / 2+3 / 4}\left(S^{T}\right)} .
\end{aligned}
$$


Extending functions $f, g, h$ by the Hestenes-Whitney method for $t>T$ we denote them by $\tilde{f}, \tilde{g}, \tilde{h}$.

Considering problem (5.4) with $\tilde{f}, \tilde{g}, \tilde{h}$ Theorem 4.6 can be applied. Hence Theorem 1 is proved.

\section{Conclusions}

In view of (5.6) the constant $c_{0}$ which appears in (1.11) depends exponentially on $T$. We eliminate the dependence on $T$ for system (1.1) with $g_{0}=0, h_{03}=0$. In this case we obtain for solutions of (1.1) the following energy estimate

$$
\begin{aligned}
& \|v(t)\|_{L_{2}(\Omega)}+\|v\|_{L_{2}\left(0, t ; H^{1}(\Omega)\right)}+\sum_{\alpha=1}^{2}\left\|v \cdot \bar{\tau}_{\alpha}\right\|_{L_{2}\left(0, t ; L_{2}(S)\right)} \\
& \leq c_{*}\left(\left\|f_{0}\right\|_{L_{2}\left(\Omega^{t}\right)}+\left\|v_{0}\right\|_{L_{2}(\Omega)}+\sum_{\alpha=1}^{2}\left\|h_{0 \alpha}\right\|_{L_{2}\left(0, t ; L_{2}(S)\right)}\right),
\end{aligned}
$$

where $t \leq T$ and $c_{*}$ is an absolute constant independent of $T$.

Using (6.1) and Theorem 3.1.1 from [17, Ch. 3] we obtain that in this case constant $c_{0}$ does not depend on $T$. To prove (6.1) the Korn inequality was used (see [19]).

Open Access This article is distributed under the terms of the Creative Commons Attribution License which permits any use, distribution, and reproduction in any medium, provided the original author(s) and the source are credited.

\section{References}

1. Agranovich, M.S., Vishik, M.I.: Elliptic problems with parameters and parabolic problem of general type. Usp. Mat. Nauk 19(117), 53-161 (1964) (in Russian)

2. Alame, W.: On existence of solutions for the nonstationary Stokes system with slip boundary conditions. Banach Center Publ. 70, 21-49 (2005)

3. Alame, W.: On the existence of solutions for the nonstationary Stokes system with slip boundary conditions. Appl. Math. 32, 195-223 (2005)

4. Besov, O.V.: Theorems of imbedding and extension of some class of functional spaces. Trudy Mat. Inst. Steklov 60, 42-81 (1961) (in Russian)

5. Besov, O.V., Il'in, V.P., Nikolskij, S.M.: Integral representation of functions and theorems of imbedding. Nauka, Moscow (1975) (in Russian); translated from Russian: Scripta Series in Mathematics, vol. 1, pp. VIII+345. New York, Toronto, Ontario, London (1978)

6. Bugrov, Ya.S.: Functional spaces with mixed norms. Izv. Akad. Nauk SSSR Ser. Mat. 35, 1137-1158 (1971)

7. Burnat, M., Zajączkowski, W.M.: On local motion of a compressible barotropic viscous fluid with the boundary slip condition. Top. Meth. Nonlinear Anal. 10, 195-223 (1997)

8. Galdi, G.P.: An introduction to the mathematical theory of the Navier-Stokes equations, vol. 1. In: Linearized Steady Problems. Springer Tracts in Natural Philosophy, vol. 38. Springer, Berlin (1994)

9. Lions, J.L., Magenes, E.: Non-Homogeneous Boundary Value Problems and Applications. Springer, Berlin (1972)

10. Ladyzhenskaya, O.A., Solonnikov, V.A., Uraltseva, N.N.: Linear and quasilinear equations of parabolic type. Nauka, Moscow (1967) (in Russian) 
11. Mucha, P., Zajączkowski, W.M.: On the existence for the Cauchy-Neumann problem for the Stokes system in the $L_{p}$-framework. Studia Math. 143(1), 75-101 (2000)

12. Solonnikov, V.A.: An initial-boundary value problem for Stokes system that arises in the study of a problem with a free boundary. Trudy Mat. Inst. Steklov 188, 150-188 (1990) (in Russian); English transl. Proc. Steklov Inst. Math. 3, 191-239 (1991)

13. Solonnikov, V.A.: Estimates of solutions of the nonstationary linearized Navier-Stokes system. Trudy MIAN SSSR 70, 213-317 (1964) (in Russian)

14. Solonnikov, V.A.: On differential properties of solutions to the first boundary value problem for the nonstationary Navier-Stokes system. Trudy MIAN SSSR 73, 221-291 (1964) (in Russian)

15. Solonnikov, V.A.: Estimates of solutions for some initial-boundary problem for the linear nonstationary Navier-Stokes system. Zap. Nauchn. Sem. LOMI 59, 178-254 (1976) (in Russian)

16. Solonnikov, V.A.: On solvability of the second initial-boundary value problem for the linear nonstationary Navier-Stokes system. Zap. Nauchn. Sem. LOMI 69, 200-218 (1977) (in Russian)

17. von Wahl, W.: The equations of Navier-Stokes equations and abstract parabolic equations. Vieweg \& Sohn, Braunschweig (1985)

18. Zajączkowski, W.M.: Nonstationary Stokes system in weighted Sobolev spaces. Math. Meth. Appl. Sci. 34, 544-562 (2011)

19. Zajączkowski, W.M.: Long time existence of regular solutions to Navier-Stokes equations in cylindrical domains under boundary slip conditions. Studia Math. 169(3), 243-285 (2005) 